Interfaces and Free Boundaries 13 (2011), 463-484

DOI $10.4171 / \mathrm{IFB} / 268$

\title{
The Muskat problem for a viscoelastic filtration
}

\author{
ANVARBEK MEIRMANOV \\ Belgorod State University, ul. Pobedy 85, 308015 Belgorod, Russia \\ E-mail: anvarbek@list.ru
}

[Received 20 May 2010 and in revised form 6 July 2011]

\begin{abstract}
A free boundary problem describing joint filtration of two immiscible incompressible liquids is derived from homogenization theory. We start with a mathematical model on the microscopic level, which consists of the stationary Stokes system for an incompressible inhomogeneous viscous liquid, occupying a pore space, the stationary Lamé equations for an incompressible elastic solid skeleton, coupled with suitable boundary conditions on the common boundary "solid skeleton - pore space", and a transport equation for the unknown liquid density. Next we prove the solvability of this model and rigorously perform the homogenization procedure as the dimensionless size of pores tends to zero, while the porous body is geometrically periodic. As a result, we prove the solvability of the Muskat problem for viscoelastic filtration.
\end{abstract}

2010 Mathematics Subject Classification: 35R35, 35M13, 35B27.

Keywords: Muskat problem; free boundary problems; liquid filtration; homogenization of periodic structures; Darcy law.

\section{Introduction}

In the present publication we consider mathematical models describing a joint motion of two immiscible liquids in porous media, for example, the displacement of oil in water. There are different types of such models, but we are interested only in some of the fundamental models of continuum mechanics, such as, for example, Stokes equations for a slow motion of a viscous liquid, or Lamé's equations for displacements of an elastic solid body, or in models asymptotically close to these.

Among the mathematical models of a joint motion of two immiscible liquids the most widely recognized one is the Muskat problem, suggested by M. Muskat [9]. This model describes filtration of two immiscible incompressible liquids of different viscosities and different densities, divided by some unknown (free) boundary. The motion of the first liquid in the domain $\Omega^{+}(t)$ with a constant viscosity $\mu^{+}$and a constant density $\rho_{f}^{+}$is governed by the Darcy system of filtration

$$
\boldsymbol{v}^{+}=-\frac{k}{\mu^{+}} \nabla p_{f}^{+}+\rho_{f}^{+} \boldsymbol{F}, \quad \nabla \cdot \boldsymbol{v}^{+}=0, \quad \boldsymbol{x} \in \Omega^{+}(t),
$$

for the macroscopic velocity $\boldsymbol{v}^{+}$and the macroscopic pressure $p_{f}^{+}$of the liquid. Correspondingly, the motion of the second liquid in the domain $\Omega^{-}(t)$ with a constant viscosity $\mu^{-}$and a constant density $\rho_{f}^{-}$is governed by the Darcy system

$$
\boldsymbol{v}^{-}=-\frac{k}{\mu^{-}} \nabla p_{f}^{-}+\rho_{f}^{-} \boldsymbol{F}, \quad \nabla \cdot \boldsymbol{v}^{-}=0, \quad \boldsymbol{x} \in \Omega^{-}(t),
$$

for the macroscopic velocity $\boldsymbol{v}^{-}$and the macroscopic pressure $p_{f}^{-}$. 
On the common free boundary $\Gamma(t)=\partial \Omega^{+}(t) \cap \partial \Omega^{-}(t)$ the pressures and normal velocities are continuous:

$$
\begin{gathered}
p_{f}^{+}=p_{f}^{-}, \quad \boldsymbol{x} \in \Gamma(t), \\
\boldsymbol{v}^{+} \cdot \boldsymbol{n}=\boldsymbol{v}^{-} \cdot \boldsymbol{n}=V_{n}, \quad \boldsymbol{x} \in \Gamma(t),
\end{gathered}
$$

where $\boldsymbol{n}$ is the unit normal vector to the boundary $\Gamma(t)$ at the point $\boldsymbol{x} \in \Gamma(t)$ and $V_{n}$ is the velocity in the normal direction to the boundary $\Gamma(t)$ at $\boldsymbol{x}$.

In $(0.1)-(0.2), k$ is the permeability coefficient of a given physical medium, and $\boldsymbol{F}$ is a dimensionless vector of distributed mass forces. These data are supposed to be known.

Condition (0.4) means that the boundary $\Gamma(t)$ is a material surface-it consists of the same set of material points during the motion. This fact permits a weak formulation of the Muskat problem. Namely, we define the pressure $p_{f}$ of the inhomogeneous liquid as

$$
p_{f}=p_{f}^{+} \quad \text { if } \boldsymbol{x} \in \Omega^{+}(t), \quad p_{f}=p_{f}^{-} \quad \text { if } \boldsymbol{x} \in \Omega^{-}(t)
$$

the density $\rho_{f}$ as

$$
\rho_{f}=\rho_{f}^{+} \quad \text { if } \boldsymbol{x} \in \Omega^{+}(t), \quad \rho_{f}=\rho_{f}^{-} \quad \text { if } \boldsymbol{x} \in \Omega^{-}(t),
$$

the viscosity $\mu$ as

$$
\mu=\mu^{+} \quad \text { if } \boldsymbol{x} \in \Omega^{+}(t), \quad \mu=\mu^{-} \quad \text { if } \boldsymbol{x} \in \Omega^{-}(t),
$$

and the velocity $\boldsymbol{v}$ as

$$
\boldsymbol{v}=\boldsymbol{v}^{+} \quad \text { if } \boldsymbol{x} \in \Omega^{+}(t), \quad \boldsymbol{v}=\boldsymbol{v}^{-} \quad \text { if } \boldsymbol{x} \in \Omega^{-}(t) .
$$

Then the unknown functions $v, p_{f}, \mu$ and $\rho_{f}$ satisfy the Darcy system of filtration in the form

$$
\boldsymbol{v}=-\frac{k}{\mu} \nabla p_{f}+\rho_{f} \boldsymbol{F}, \quad \nabla \cdot \boldsymbol{v}=0, \quad \boldsymbol{x} \in \Omega, t>0,
$$

and the transport equations

$$
\begin{array}{ll}
\frac{\mathrm{d} \rho_{f}}{\mathrm{~d} t} \equiv \frac{\partial \rho_{f}}{\partial t}+\boldsymbol{v} \cdot \nabla \rho_{f}=0, & x \in \Omega, t>0, \\
\frac{\mathrm{d} \mu}{\mathrm{d} t} \equiv \frac{\partial \mu}{\partial t}+\boldsymbol{v} \cdot \nabla \mu=0, & \boldsymbol{x} \in \Omega, t>0 .
\end{array}
$$

The first equation in (0.5) (Darcy law) is understood in the usual sense almost everywhere in $\Omega_{T}=$ $\Omega \times(0, T)$, and the second equation (continuity equation) is understood in the sense of distributions. The transport equations are understood in the sense of distributions, if we use the equalities

$$
\boldsymbol{v} \cdot \nabla \mu=\nabla \cdot(\boldsymbol{v} \mu), \quad \boldsymbol{v} \cdot \nabla \rho_{f}=\nabla \cdot\left(\boldsymbol{v} \rho_{f}\right) .
$$

The problem is complemented with the homogeneous boundary condition

$$
\boldsymbol{v} \cdot \boldsymbol{n}=0, \quad \boldsymbol{x} \in S=\partial \Omega, t>0,
$$


where $\boldsymbol{n}$ is the normal vector to the boundary $S$, and the initial conditions

$$
\rho_{f}(\boldsymbol{x}, 0)=\rho_{f}^{0}(\boldsymbol{x}), \quad \mu(\boldsymbol{x}, 0)=\mu_{0}(\boldsymbol{x}), \quad \boldsymbol{x} \in \Omega,
$$

with discontinuous initial data:

$$
\rho_{f}^{0}(\boldsymbol{x})=\rho_{f}^{+}=\mathrm{const}>0, \quad \mu_{0}(\boldsymbol{x})=\mu^{+}=\mathrm{const}>0, \quad \boldsymbol{x} \in \Omega^{+},
$$

and

$$
\rho_{f}^{0}(\boldsymbol{x})=\rho_{f}^{-}=\mathrm{const}>0, \quad \mu_{0}(\boldsymbol{x})=\mu^{-}=\mathrm{const}>0, \quad \boldsymbol{x} \in \Omega^{-},
$$

where $\Gamma(0)=\partial \Omega^{-} \cap \partial \Omega^{+}$is a sufficiently smooth surface.

So, one has two settings of the same Muskat problem. In both cases the problem is easy to formulate, but almost impossible to solve. For this reason, very little is known about classical or weak solutions. There are only a few results on classical solvability locally in time or globally in time, but no explicit solutions, and there is no result on weak solvability (see [16], [12], [14] and references there).

The goal of this paper is to find more general mathematical models describing the same physical process and asymptotically close to some fundamental models of continuum mechanics. To explain our ideas we consider for the moment only the Darcy system of filtration, which is responsible for the dynamics in the Muskat problem. It is well-known that this system is an asymptotic limit of the Stokes system for an incompressible viscous liquid, when the dimensionless pore size tends to zero (see [2], [13]). But this Stokes system on the microscopic level is a particular case $\left(\alpha_{\tau}=0\right.$, $\left.\alpha_{\lambda}=\infty\right)$ of a more general system

$$
\begin{gathered}
\alpha_{\tau} \rho^{\varepsilon} \frac{\partial^{2} \boldsymbol{w}}{\partial t^{2}}=\nabla \cdot\left(\chi^{\varepsilon} \alpha_{\mu} \mathbb{D}\left(x, \frac{\partial \boldsymbol{w}}{\partial t}\right)+\left(1-\chi^{\varepsilon}\right) \alpha_{\lambda} \mathbb{D}(x, \boldsymbol{w})-p \mathbb{I}\right)+\rho^{\varepsilon} \boldsymbol{F}, \\
\nabla \cdot \boldsymbol{w}=0,
\end{gathered}
$$

for the displacement $\boldsymbol{w}$ and the pressure $p$ of continuous medium ([2], [8], [11], [13]). The microscopic system $(0.10),(0.11)$ describes the joint motion of the viscous liquid in a pore space and of an elastic solid skeleton and is understood in the sense of distributions. Roughly speaking, this system contains the Stokes system for the viscous liquid in the pore space, the Lamé system for the solid skeleton and the boundary condition (the continuity of the normal stresses) on the common boundary "solid skeleton - pore space".

In $(0.10), \mathbb{D}(x, \boldsymbol{w})$ is the symmetric part of $\nabla \boldsymbol{w}, \chi^{\varepsilon}$ is the characteristic function of the pore space, $\varepsilon=l / L$ is the dimensionless pore size,

$$
\alpha_{\tau}=\frac{L}{g \tau^{2}}, \quad \alpha_{\mu}=\frac{2 \mu}{\tau L g \rho_{0}}, \quad \alpha_{\lambda}=\frac{2 \lambda}{L g \rho_{0}}, \quad \rho^{\varepsilon}=\rho_{f} \chi^{\varepsilon}+\rho_{s}\left(1-\chi^{\varepsilon}\right),
$$

$l$ is an average size of pore, $L$ is a characteristic size of the domain under consideration, $\tau$ is a characteristic time of the process, $\rho_{f}$ and $\rho_{s}$ are the respective mean dimensionless densities of the liquid in pores and the solid skeleton correlated with the mean density of water $\rho_{0}, g$ is the acceleration of gravity, $\mu$ is the viscosity of the liquid, and $\lambda$ is the elastic Lamé constant.

Theoretically the microscopic system $(0.10),(0.11)$ with appropriate initial and boundary conditions is one of the most suitable mathematical models, describing the joint motion of a viscous 
liquid in a pore space and an elastic solid skeleton. But this model has no practical significance, since it is necessary to solve the problem in the physical scale of a few hundred meters, while the coefficients oscillate on the scale of a few tens of microns. The practical significance of the model appears only after homogenization. So, we have to let all dimensionless criteria $\alpha_{\tau}, \alpha_{\mu}$ and $\alpha_{\lambda}$ to be variable functions, depending on a small parameter $\varepsilon$, and find all limiting regimes as $\varepsilon \rightarrow 0$.

First of all, note that in the present paper we consider only filtration processes, where the characteristic time $\tau$ of the processes is about several months. Then

$$
\alpha_{\tau} \rightarrow 0 \quad \text { as } \varepsilon \rightarrow 0
$$

and we may neglect the inertial term in the dynamic equation $(0.10)$ and rewrite it in the form

$$
\nabla \cdot\left(\chi^{\varepsilon} \alpha_{\mu} \mathbb{D}\left(x, \frac{\partial \boldsymbol{w}}{\partial t}\right)+\left(1-\chi^{\varepsilon}\right) \alpha_{\lambda} \mathbb{D}(x, \boldsymbol{w})-p \mathbb{I}\right)+\rho^{\varepsilon} \boldsymbol{F}=0 .
$$

Next we note that for almost all physical processes, $\alpha_{\mu} \sim 0$ and $\alpha_{\lambda}$ is sufficiently large. Then the asymptotic limit of (0.11), (0.12) under the conditions

$$
\alpha_{\mu} \sim O\left(\varepsilon^{2}\right), \quad \alpha_{\lambda} \rightarrow \infty \quad \text { as } \varepsilon \searrow 0
$$

is the Darcy system of filtration. We say that the Darcy system of filtration is the first level approximation of the microscopic system (0.11), (0.12). The second level approximation of (0.11), (0.12) is the Terzaghi-Biot system of poroelasticity ([1], [15]) and corresponds to the conditions

$$
\alpha_{\mu} \sim O\left(\varepsilon^{2}\right), \quad \alpha_{\lambda} \sim O(1)
$$

Finally, even for sufficiently small $\alpha_{\mu}$ and sufficiently large $\alpha_{\lambda}$ we may always suppose that

$$
0<\lambda_{0}, \mu_{0}<\infty
$$

where

$$
\lambda_{0}=\lim _{\varepsilon \searrow 0} \alpha_{\lambda}, \quad \mu_{0}=\lim _{\varepsilon \searrow 0} \alpha_{\mu} .
$$

After a homogenization procedure we arrive at equations of viscoelastic filtration ([2], [8], [11]), which are the third level approximation of $(0.11),(0.12)$.

It is clear that all different asymptotic models of the system $(0.11),(0.12)$ describe the same physical process, but with different degrees of approximation. The choice of the model depends on the aims of the researcher.

The same method can be applied to the Muskat problem. On the microscopic level the free boundary problem consists of the initial boundary value problem for dynamic equations $(0.11)$, (0.12) for the displacement $\boldsymbol{w}$ and the pressure $p$ of the continuous medium, coupled with the Cauchy problem

$$
\begin{aligned}
\frac{\partial \rho_{f}}{\partial t}+\frac{\partial \boldsymbol{w}}{\partial t} \cdot \nabla \rho_{f} & =0, & \rho_{f}(\boldsymbol{x}, 0) & =\rho_{f}^{(0)}(\boldsymbol{x}), \\
\frac{\partial \mu}{\partial t}+\frac{\partial \boldsymbol{w}}{\partial t} \cdot \nabla \mu & =0, & \mu(\boldsymbol{x}, 0) & =\mu_{0}(\boldsymbol{x})
\end{aligned}
$$

for the viscosity $\mu$ and the density $\rho_{f}$ of the inhomogeneous liquid in the pore space. 
To make all ideas clear, we consider the simplest case of the problem when

$$
\mu^{+}=\mu^{-}=\mu .
$$

Then the first level approximation of the microscopic free boundary problem is the Muskat problem, which consists of the dynamic equations in the form of the Darcy system of filtration

$$
\boldsymbol{v}=-\frac{1}{\mu_{1}} \mathbb{B}^{(f)} \cdot\left(\nabla p_{f}+\rho_{f} \boldsymbol{F}\right), \quad \nabla \cdot \boldsymbol{v}=0,
$$

for the velocity $\boldsymbol{v}$ and the pressure $p_{f}$ of the liquid with a symmetric strictly positive definite matrix $\mathbb{B}^{(f)}$, and the transport equation $(0.6)$ for the density $\rho_{f}$. In (0.15) the criterion

$$
\mu_{1}=\lim _{\varepsilon \searrow 0} \frac{\alpha_{\mu}}{\varepsilon^{2}}
$$

is strictly positive and finite.

The second level approximation of the microscopic free boundary problem $(0.11),(0.12),(0.14)$ will be called the Muskat-Terzaghi-Biot problem. This problem consists of the transport equation (0.6) for the density of the liquid $\rho_{f}$ and the Terzaghi-Biot system of poroelasticity

$$
\begin{gathered}
\boldsymbol{v}=m \frac{\partial \boldsymbol{u}}{\partial t}-\frac{1}{\mu_{1}} \mathbb{B}^{(f)} \cdot\left(\nabla p_{f}+\rho_{f} \boldsymbol{F}\right), \quad \nabla \cdot\left(\boldsymbol{v}+(1-m) \frac{\partial \boldsymbol{u}}{\partial t}\right)=0, \\
\nabla \cdot\left(\lambda_{0} \mathbb{A}_{0}^{s}: \mathbb{D}(x, \boldsymbol{u})-\frac{1}{m} p_{f} \mathbb{I}\right)+\rho \boldsymbol{F}=0,
\end{gathered}
$$

for the velocity $\boldsymbol{v}$ and the pressure $p_{f}$ of the liquid and the displacement $\boldsymbol{u}$ of the solid skeleton. In (0.16), (0.17), $m$ is the porosity of the pore space, $\rho=m \rho_{f}-(1-m) \rho_{s}$ is the density of the mixture of the solid skeleton and the liquid in pores, $\mathbb{A}_{0}^{s}$ is a symmetric strictly positive definite constant fourth-rank tensor (for the definition of $\mathbb{A}_{0}^{s}$ and $\mathbb{B}^{(f)}$ see [8]) and the criterion $\lambda_{0}$ is strictly positive and finite.

The last model has a submodel, corresponding to the case $\mu_{1}=\infty$. This submodel consists of the dynamic system

$$
\nabla \cdot\left(\lambda_{0} \mathbb{A}_{0}^{s}: \mathbb{D}(x, \boldsymbol{u})-\frac{1}{m} p_{f} \mathbb{I}\right)+\rho \boldsymbol{F}=0, \quad \nabla \cdot \boldsymbol{u}=0,
$$

for the solid displacement $\boldsymbol{u}$ and the liquid pressure $p_{f}$, and the transport equation (0.6) for the liquid density $\rho_{f}$, where the liquid velocity is defined by

$$
\boldsymbol{v}=m \frac{\partial \boldsymbol{u}}{\partial t} .
$$

This relation formally follows from the first equation in (0.16) if we put there $\mu_{1}=\infty$. We call this problem the Muskat problem for elastic filtration.

Finally, the third level approximation of the microscopic free boundary problem $(0.11),(0.12)$, $(0.14)$ is the Muskat problem for viscoelastic filtration. This problem consists of the dynamic system of viscoelastic filtration

$$
\begin{gathered}
\nabla \cdot\left(\mathbb{P}^{(0)}(\boldsymbol{w})\right)-\nabla p+\rho \boldsymbol{F}=0, \quad \nabla \cdot \boldsymbol{w}=0 \\
\mathbb{P}^{(0)}(\boldsymbol{w})=\mathbb{A}_{0}: \mathbb{D}\left(x, \frac{\partial \boldsymbol{w}}{\partial t}\right)+\mathbb{A}_{1}: \mathbb{D}(x, \boldsymbol{w})+\int_{0}^{t} \mathbb{A}_{2}(t-\tau): \mathbb{D}(x, \boldsymbol{w}(\boldsymbol{x}, \tau)) \mathrm{d} \tau
\end{gathered}
$$


for the displacement $\boldsymbol{w}$ and the pressure $p$ of the mixture of the liquid and solid phases and equation (0.6) for the liquid density $\rho_{f}$ with $\boldsymbol{v}=\partial \boldsymbol{w} / \partial t$. Note that this transport equation may be rewritten as the transport equation

$$
\frac{\partial \rho}{\partial t}+\boldsymbol{v} \cdot \nabla \rho=0
$$

for the density $\rho$ of the mixture.

To prove the well-posedness of the above mentioned free boundary problems on the macroscopic level we must

1) prove the existence of a weak solution $\left\{\boldsymbol{w}^{\varepsilon}, p^{\varepsilon}, \rho_{f}^{\varepsilon}\right\}$ to the free boundary problem $(0.11),(0.12)$, (0.14) on the microscopic level for every fixed $\varepsilon>0$,

2) perform the rigorous homogenization procedure as $\varepsilon \searrow 0$.

In the present paper we do it for the Muskat problem for viscoelastic filtration under condition (0.13). The main problem here is the limiting procedure in the nonlinear term $\boldsymbol{v}^{\varepsilon} \rho_{f}^{\varepsilon}\left(\boldsymbol{v}^{\varepsilon}=\partial \boldsymbol{w}^{\varepsilon} / \partial t\right)$ in the transport equation. Note that the liquid density $\rho_{f}^{\varepsilon}$ is just a bounded (and discontinuous) function. So, we may only expect the weak convergence of the sequence $\left\{\rho_{f}^{\varepsilon}\right\}$ in $L_{2}\left(\Omega_{T}\right)$. Therefore, we have to prove the strong convergence of the sequence $\left\{\boldsymbol{v}^{\varepsilon}\right\}$ in $L_{2}\left(\Omega_{T}\right)$, and this task becomes crucial.

Notations for function spaces and norms are the same as in [6] and [7].

\section{The problem statement and main results}

First of all let us describe the domain $\Omega$, a solid skeleton and a pore space. We suppose that $\Omega$ is a unit cube and obtained by periodic repetition of an elementary cell $\varepsilon Y$. In turn, the open unit cube $Y=Y_{f} \cup \gamma \cup Y_{s}$ models a pore space, a solid skeleton, and a solid-liquid interface, such that the connected pore space $\Omega_{f}^{\varepsilon}$ is the periodic repetition of the elementary cell $\varepsilon Y_{f}$, the solid skeleton $\Omega_{s}^{\varepsilon}$ is the periodic repetition of the elementary cell $\varepsilon Y_{s}$, and the Lipschitz continuous solid-liquid interface $S^{(\varepsilon)}$ is the periodic repetition of the Lipschitz continuous interface $\varepsilon \gamma$. To avoid many technical problems, connected with a priori estimates of the solutions, independent of a small parameter $\varepsilon$, we assume that the solid skeleton is disconnected. That is, the elementary solid cell $Y_{s}$ is completely surrounded by the elementary liquid cell $Y_{f}$ and $\gamma \cap \partial Y=\emptyset$.

To make all proofs clearer we suppose that $1 / \varepsilon$ is an integer, so that always $\partial \Omega_{s}^{\varepsilon} \cap \partial \Omega=\emptyset$.

Next we have to complete the Cauchy problem for the transport equation $(0.14)$. On the microscopic level the transport equation for the liquid density is defined only in the pore space $\Omega_{f}^{\varepsilon}$. For the exact microscopic model (see [8]) the characteristic function $\tilde{\chi}^{\varepsilon}$ of the pore space is an unknown function and is defined as a solution to the Cauchy problem for the transport equation

$$
\frac{\partial \tilde{\chi}^{\varepsilon}}{\partial t}+\frac{\partial \boldsymbol{w}}{\partial t} \cdot \nabla \tilde{\chi}^{\varepsilon}=0, \quad \tilde{\chi}^{\varepsilon}(\boldsymbol{x}, 0)=\chi^{\varepsilon}(\boldsymbol{x})
$$

It means that the solid-liquid interface $S^{(\varepsilon)}$ in the exact model is a material surface and we do not need boundary conditions for the liquid density on $S^{(\varepsilon)}$. But our basic dynamic system here is a linear system, where $\tilde{\chi}^{\varepsilon}=\chi^{\varepsilon}$ is a given function. Therefore the solid-liquid interface $S^{(\varepsilon)}$ is not a material surface anymore and we need a boundary condition for the liquid density on the part of $S^{(\varepsilon)}$ where the liquid "enters" the pore space. To avoid this, we extend the Cauchy problem $(0.14)$ onto 
the whole domain $\Omega$. First we suppose that the function $\rho_{f}^{(0)}(\boldsymbol{x})$ is defined in $\Omega$ and

$$
\rho_{f}^{(0)}(\boldsymbol{x})=\rho_{f}^{+} \quad \text { in } \Omega^{+}, \quad \rho_{f}^{(0)}(\boldsymbol{x})=\rho_{f}^{-} \quad \text { in } \Omega^{-}, \quad \bar{\Omega}=\overline{\Omega^{+}} \cup \overline{\Omega^{-}} .
$$

Now let us look at the dynamic equation (0.12). The smoothness of the solution with respect to time in the solid part depends on the smoothness of the term $\rho^{\varepsilon} \boldsymbol{F}$ with respect to time. But $\rho^{\varepsilon}$ might be some step function, which does not guarantee the existence of $\partial \boldsymbol{w} / \partial t$. Therefore, instead of $\partial \boldsymbol{w} / \partial t$ we have to consider some function $\boldsymbol{v}$, which coincides with $\partial \boldsymbol{w} / \partial t$ in the liquid part and is asymptotically close to $\partial \boldsymbol{w} / \partial t$ as $\varepsilon \rightarrow 0$ in the solid part.

To do that, we extend the liquid velocity $\partial \boldsymbol{w} / \partial t$ from the liquid part $\Omega_{f}^{\varepsilon}$ to the solid part $\Omega_{s}^{\varepsilon}$ using the extension result of C. Conca [3]: for any $\varepsilon>0$ there exists an extension $\boldsymbol{v}(\boldsymbol{x}, t) \in \mathscr{W}_{2}^{1}(\Omega)$ of the function $(\partial \boldsymbol{w} / \partial t)(\boldsymbol{x}, t)$ from $\Omega_{f}^{\varepsilon}$ onto $\Omega$ such that

$$
\chi^{\varepsilon}(\boldsymbol{x})\left(\boldsymbol{v}(\boldsymbol{x}, t)-\frac{\partial \boldsymbol{w}}{\partial t}(\boldsymbol{x}, t)\right)=0, \quad \boldsymbol{x} \in \Omega, t>0,
$$

and

$$
\int_{\Omega}|\boldsymbol{v}|^{2} \mathrm{~d} x \leqslant C \int_{\Omega_{f}^{\varepsilon}}\left|\frac{\partial \boldsymbol{w}}{\partial t}\right|^{2} \mathrm{~d} x, \quad \int_{\Omega}|\mathbb{D}(x, \boldsymbol{v})|^{2} \mathrm{~d} x \leqslant C \int_{\Omega_{f}^{\varepsilon}}\left|\mathbb{D}\left(x, \frac{\partial \boldsymbol{w}}{\partial t}\right)\right|^{2} \mathrm{~d} x,
$$

where $C$ is independent of $\varepsilon$ and $t$. In the present paper we fix this continuous linear operator from $W_{2}^{1}\left(\Omega_{f}^{\varepsilon}\right)$ to $W_{2}^{1}(\Omega)$ and denote it as

$$
\boldsymbol{v}=\mathbb{E}_{\Omega_{f}^{\varepsilon}}\left(\frac{\partial \boldsymbol{w}}{\partial t}\right),
$$

and, in what follows, call this function the liquid velocity.

Note that due to the continuity equation (0.11) in the domain $\Omega_{T}$ and the structure of the pore space we may choose the extension operator such that the function $\boldsymbol{v}$ will be solenoidal:

$$
\nabla \cdot \boldsymbol{v}=0, \quad(\boldsymbol{x}, t) \in \Omega_{T} .
$$

For more details see [5].

Finally, we rewrite the transport equation for the liquid density as an equation for the density of the mixture

$$
\rho=\chi^{\varepsilon} \rho_{f}+\left(1-\chi^{\varepsilon}\right) \rho_{s}
$$

in the form

$$
\frac{\partial \rho}{\partial t}+\boldsymbol{v} \cdot \nabla \rho=0, \quad(\boldsymbol{x}, t) \in \Omega_{T} ; \quad \rho(\boldsymbol{x}, 0)=\rho^{(0)}(\boldsymbol{x}), \quad \boldsymbol{x} \in \Omega,
$$

where

and $\boldsymbol{v}$ is defined by (1.2).

$$
\rho^{(0)}(\boldsymbol{x})=\chi^{\varepsilon} \rho_{f}^{(0)}(\boldsymbol{x})+\left(1-\chi^{\varepsilon}\right) \rho_{s}
$$

Now we are ready to formulate the definition of a weak solution to the free boundary problem on the microscopic level. 
We say that a triple $\left\{\boldsymbol{w}^{\varepsilon}, p^{\varepsilon}, \rho^{\varepsilon}\right\}$ of functions is a weak solution to the problem $(\mathbf{F B})^{(\varepsilon)}$ if $\boldsymbol{w}^{\varepsilon}$ is solenoidal in $\Omega_{T}=\Omega \times(0, T)$,

$$
\begin{gathered}
\boldsymbol{w}^{\varepsilon} \in L_{2}\left((0, T) ; \stackrel{\circ}{W}_{2}^{1}(\Omega)\right), \quad \chi^{\varepsilon} \nabla\left(\frac{\partial \boldsymbol{w}^{\varepsilon}}{\partial t}\right) \in L_{2}\left(\Omega_{T}\right), \quad \rho^{\varepsilon} \in L_{\infty}\left(\Omega_{T}\right), \\
p^{\varepsilon} \in L_{2}\left(\Omega_{T}\right), \quad \int_{\Omega} p^{\varepsilon}(\boldsymbol{x}, t) \mathrm{d} x=0,
\end{gathered}
$$

and we have the integral identities

$$
\int_{\Omega_{T}} \rho^{\varepsilon}\left(\frac{\partial \xi}{\partial t}+\boldsymbol{v}^{\varepsilon} \cdot \nabla \xi\right) \mathrm{d} x \mathrm{~d} t+\int_{\Omega} \rho^{(0)}(\boldsymbol{x}) \xi(\boldsymbol{x}, 0) \mathrm{d} x=0
$$

and

$$
\begin{aligned}
\int_{\Omega_{T}}\left(-\chi^{\varepsilon} \alpha_{\mu} \mathbb{D}\left(x, \boldsymbol{w}^{\varepsilon}\right): \mathbb{D}\left(x, \frac{\partial \boldsymbol{\varphi}}{\partial t}\right)+\left(1-\chi^{\varepsilon}\right) \alpha_{\lambda}\right. & \left.\mathbb{D}\left(x, \boldsymbol{w}^{\varepsilon}\right): \mathbb{D}(x, \boldsymbol{\varphi})\right) \mathrm{d} x \mathrm{~d} t \\
& =\int_{\Omega_{T}}\left(p^{\varepsilon} \nabla \cdot \boldsymbol{\varphi}+\rho^{\varepsilon} \boldsymbol{F} \cdot \boldsymbol{\varphi}\right) \mathrm{d} x \mathrm{~d} t,
\end{aligned}
$$

for any smooth functions $\xi$ and $\varphi$ such that $\xi(x, T)=0$ and $\varphi(x, t)=0$ for $\boldsymbol{x} \in S$.

In (1.4), $\boldsymbol{v}^{\varepsilon}$ is an extension of $\partial \boldsymbol{w}^{\varepsilon} / \partial t$ given by (1.2) and

$$
\boldsymbol{v}^{\varepsilon} \in L_{2}\left((0, T) ; \stackrel{\circ}{W}_{2}^{1}(\Omega)\right)
$$

because $1 / \varepsilon$ is an integer.

Note that the integral identity (1.5) contains differential equations in the pore space and in the solid skeleton, a boundary condition on $S^{(\varepsilon)}$ and the initial condition

$$
\chi^{\varepsilon}(\boldsymbol{x}) \boldsymbol{w}^{\varepsilon}(\boldsymbol{x}, 0)=0 .
$$

THEOREM 1 Suppose that

$$
\max _{(\boldsymbol{x}, t) \in \Omega_{T}}\left(|\boldsymbol{F}(\boldsymbol{x}, t)|+|\nabla \boldsymbol{F}(\boldsymbol{x}, t)|+\left|\frac{\partial \boldsymbol{F}}{\partial t}(\boldsymbol{x}, t)\right|\right)=F<\infty,
$$

and condition $(0.13)$ holds true. Then the problem $(\mathbf{F B})^{\varepsilon}$ has a weak solution $\left\{\boldsymbol{w}^{\varepsilon}, p^{\varepsilon}, \rho^{\varepsilon}\right\}$ such that

$$
\begin{gathered}
\max _{0<t<T} \int_{\Omega}\left(\left|\boldsymbol{w}^{\varepsilon}\right|^{2}+\left|\boldsymbol{v}^{\varepsilon}\right|^{2}+\left|\nabla \boldsymbol{w}^{\varepsilon}(\boldsymbol{x}, t)\right|^{2}\right) \mathrm{d} x+\int_{\Omega_{T}}\left|p^{\varepsilon}\right|^{2} \mathrm{~d} x \mathrm{~d} t \leqslant C, \\
\max _{0<t<T} \int_{\Omega}\left(\left|\nabla \boldsymbol{v}^{\varepsilon}\right|^{2}+\chi^{\varepsilon}\left|\mathbb{D}\left(x, \frac{\partial \boldsymbol{w}^{\varepsilon}}{\partial t}(\boldsymbol{x}, t)\right)\right|^{2}\right) \mathrm{d} x+\int_{\Omega_{T}}\left|\nabla \frac{\partial \boldsymbol{w}^{\varepsilon}}{\partial t}\right|^{2} \mathrm{~d} x \mathrm{~d} t \leqslant C, \\
\int_{\Omega}\left|\nabla \boldsymbol{v}^{\varepsilon}\left(\boldsymbol{x}, t_{1}\right)-\nabla \boldsymbol{v}^{\varepsilon}\left(\boldsymbol{x}, t_{2}\right)\right|^{2} \mathrm{~d} x \leqslant C\left|t_{1}-t_{2}\right|^{1 / 2}, \quad t_{1}, t_{2} \in(0, T), \\
0 \leqslant \rho^{\varepsilon}(\boldsymbol{x}, t) \leqslant \max \left(\rho_{f}^{-}, \rho_{f}^{+}, \rho_{s}\right)=\rho_{0}^{+},
\end{gathered}
$$

where $C=C\left(\rho_{0}^{+}, F, T\right)$ is independent of $\varepsilon$, and

$$
\boldsymbol{v}^{\varepsilon}=\mathbb{E}_{\Omega_{f}^{\varepsilon}}\left(\frac{\partial \boldsymbol{w}^{\varepsilon}}{\partial t}\right)
$$


THEOREM 2 Under the assumptions of Theorem 1 let $\left\{\boldsymbol{w}^{\varepsilon}, p^{\varepsilon}, \rho^{\varepsilon}\right\}$ be a solution to the problem $(\mathbf{F B})^{\varepsilon}$.

(I) There exists a subsequence of small parameters $\varepsilon \searrow 0$ such that

(1) $\left\{\boldsymbol{w}^{\varepsilon}\right\}$ converges weakly in $L_{2}\left((0, T) ; \stackrel{\circ}{W}_{2}^{1}(\Omega)\right)$ and strongly in $L_{2}\left(\Omega_{T}\right)$ to a function $\boldsymbol{w}$,

(2) $\left\{\rho^{\varepsilon}\right\}$ converges weakly in $L_{2}\left(\Omega_{T}\right)$ to a function $\rho$,

(3) $\left\{p^{\varepsilon}\right\}$ converges weakly in $L_{2}\left(\Omega_{T}\right)$ to a function $p$,

(4) $\left\{\partial \boldsymbol{w}^{\varepsilon} / \partial t\right\}$ converges weakly in $L_{2}\left((0, T) ; \stackrel{\circ}{W}_{2}^{1}(\Omega)\right)$ and strongly in $L_{2}\left(\Omega_{T}\right)$ to $\partial \boldsymbol{w} / \partial t$,

(5) $\left\{\boldsymbol{v}^{\varepsilon}\right\}$, where $\boldsymbol{v}^{\varepsilon}=\mathbb{E}_{\Omega_{f}^{\varepsilon}}(\partial \boldsymbol{w} / \partial t)$, converges strongly in $L_{2}\left(\Omega_{T}\right)$ to $\partial \boldsymbol{w} / \partial t$.

(II) The triple $\{\boldsymbol{w}, p, \rho\}$ of limiting functions is a weak solution to the Muskat problem for viscoelastic filtration, which consists of the dynamic equations

$$
\begin{gathered}
\nabla \cdot\left(\mathbb{P}^{(0)}(\boldsymbol{w})\right)-\nabla p+\rho \boldsymbol{F}=0, \\
\mathbb{P}^{(0)}(\boldsymbol{w})=\mathbb{A}_{0}: \mathbb{D}\left(x, \frac{\partial \boldsymbol{w}}{\partial t}\right)+\mathbb{A}_{1}: \mathbb{D}(x, \boldsymbol{w})+\int_{0}^{t} \mathbb{A}_{2}(t-\tau): \mathbb{D}(x, \boldsymbol{w}(\boldsymbol{x}, \tau) \mathrm{d} \tau, \\
\nabla \cdot \boldsymbol{w}=0,
\end{gathered}
$$

for the displacement $\boldsymbol{w}$ and the pressure $p$ of the mixture of the solid skeleton and the liquid in pores, and the transport equation

$$
\frac{\partial \rho}{\partial t}+\nabla \cdot\left(\frac{\partial \boldsymbol{w}}{\partial t} \rho\right)=0
$$

for the density $\rho$ of the mixture in the domain $\Omega_{T}$.

The problem is complemented with the homogeneous boundary condition

$$
\boldsymbol{w}(\boldsymbol{x}, t)=0, \quad \boldsymbol{x} \in S, t>0,
$$

and the initial conditions

$$
\boldsymbol{w}(\boldsymbol{x}, 0)=0, \quad \rho(\boldsymbol{x}, 0)=\rho^{(0)}(\boldsymbol{x}), \quad \boldsymbol{x} \in \Omega .
$$

In (1.10) the fourth-rank constant tensors $\mathbb{A}_{0}, \mathbb{A}_{1}$ and the fourth-rank tensor $\mathbb{A}_{2}(t)$ are defined by formulas (3.7)-(3.9) below, and the tensor $\mathbb{A}_{0}$ is symmetric and strictly positive definite.

\section{Proof of Theorem 1}

We prove the existence of a solution to the problem $(\mathbf{F B})^{\varepsilon}$ using the Schauder fixed point theorem, mollifiers, and viscosity solutions. For the correct limiting procedure we have to derive a priori estimates, independent of the parameters of approximation. First of all we approximate the density in the dynamic equation using a mollifier with respect to time. This gives us additional smoothness of the solutions to the dynamic equations with respect to time. Next we approximate the transport equation for the density by a diffusion-convection equation with a small diffusion (viscosity). This gives us additional smoothness of the density with respect to time and the spatial variables. Then we prove the existence of a solution $\left\{\rho_{(\delta, h)}, \boldsymbol{u}_{(\delta, h)}, p_{(\delta, h)}\right\}$ to the double approximate problem using the Schauder fixed point theorem. To pass to the limit as $\delta \rightarrow 0$ we need estimates independent 
of $\delta$ (Lemma 2.2). Here we essentially use the uniform boundedness of $\partial \rho_{(\delta, h)} / \partial t$ in the dual space $L_{2}\left((0, T) ; W_{2}^{-1}(\Omega)\right)$ and we also prove that these estimates are independent of $h$ and $\varepsilon$. As mentioned above, the smoothness of the solution must provide the convergence (at least weak) of the product $\rho_{(\delta, h)} \cdot \boldsymbol{v}_{(\delta, h)}$, where $\boldsymbol{v}_{(\delta, h)}=\mathbb{E}_{\Omega_{f}^{\varepsilon}}\left(\partial \boldsymbol{u}_{(\delta, h)} / \partial t\right)$. For a fixed $h>0$ the sequence $\left\{\rho_{(\delta, h)}\right\}$ is compact in $L_{2}\left(\Omega_{T}\right)$ and the sequence $\left\{\boldsymbol{v}_{(\delta, h)}\right\}$ is weakly compact in $L_{2}\left(\Omega_{T}\right)$. Thus, we may pass to the limit as $\delta \rightarrow 0$ and get a solution $\left\{\rho_{(h)}, \boldsymbol{u}_{(h)}, p_{(h)}\right\}$ of the approximate problem. As a last step we have to pass to the limit as $h \rightarrow 0$. But the bounded sequence $\left\{\rho_{(h)}\right\}$ is no longer a compact set in $L_{2}\left(\Omega_{T}\right)$. Therefore, we must prove the strong compactness of $\left\{\boldsymbol{v}_{(h)}\right\}$ in $L_{2}\left(\Omega_{T}\right)$. The functions $\boldsymbol{v}_{(h)}$ have spatial derivatives uniformly bounded in $L_{2}\left(\Omega_{T}\right)$. So, $\left\{\boldsymbol{v}_{(h)}\right\}$ is a compact set in $L_{2}(\Omega)$ for any fixed $t \in(0, T)$. To deduce that $\left\{\boldsymbol{v}_{(h)}\right\}$ is compact in $L_{2}\left(\Omega_{T}\right)$ one needs some smoothness of $\boldsymbol{v}_{(h)}$ with respect to time ([7]). But the proper dynamic equations do not provide directly this smoothness and we must find another way, which is done in Lemma 2.2.

The proof of the theorem is divided into several independent steps.

First we solve the double approximate problem

$$
\begin{gathered}
\nabla \cdot\left(\mathbb{P}^{(\varepsilon)}(\boldsymbol{u})\right)-\nabla p+(\rho)_{(\delta)} \boldsymbol{F}=0, \quad \nabla \cdot \boldsymbol{u}=0, \\
\frac{\partial \rho}{\partial t}+\boldsymbol{v} \cdot \nabla \rho=h \Delta \rho, \\
\left.\boldsymbol{u}\right|_{S}=0,\left.\quad \chi^{\varepsilon} \boldsymbol{u}\right|_{t=0}=0,\left.\quad \rho\right|_{S}=0,\left.\quad \rho\right|_{t=0}=\rho_{h}^{(0)}(\boldsymbol{x}), \\
\rho_{h}^{(0)} \in \stackrel{\circ}{C}^{\infty}(\Omega), \quad 0 \leqslant \rho_{h}^{(0)} \leqslant \rho_{0}^{+}, \quad \rho_{h}^{(0)} \rightarrow \rho^{(0)} \quad \text { a.e. in } \Omega,
\end{gathered}
$$

for $h>0$ and $\delta>0$ in $\Omega_{T}$, and then pass to the limit as $\delta \rightarrow 0$ and $h \rightarrow 0$.

In (2.1), (2.2),

$$
\begin{gathered}
\mathbb{P}^{(\varepsilon)}(\boldsymbol{u})=\chi^{\varepsilon} \alpha_{\mu} \mathbb{D}\left(x, \frac{\partial \boldsymbol{u}}{\partial t}\right)+\left(1-\chi^{\varepsilon}\right) \alpha_{\lambda} \mathbb{D}(x, \boldsymbol{u}), \\
(\rho)_{(\delta)}=\frac{1}{\delta} \int_{t-\delta}^{t} \hat{\rho}(\boldsymbol{x}, \tau) \mathrm{d} \tau, \quad \boldsymbol{v}=\mathbb{E}_{\Omega_{f}^{\varepsilon}}\left(\frac{\partial \boldsymbol{u}}{\partial t}\right), \\
\hat{\rho}(\boldsymbol{x}, t)=\rho(\boldsymbol{x}, t) \quad \text { for } t>0 \quad \text { and } \quad \hat{\rho}(\boldsymbol{x}, t)=\rho_{h}^{(0)}(\boldsymbol{x}) \quad \text { for } t<0 .
\end{gathered}
$$

By the properties of mollifiers the function $(\rho)_{(\delta)}$ possesses time derivative $\partial\left((\rho)_{(\delta)}\right) / \partial t \in L_{2}\left(\Omega_{T}\right)$. This fact guarantees the additional smoothness of the solution $\boldsymbol{u}$ with respect to time.

In a standard way we may define a weak solution to the problem $(2.1)-(2.3)$ as functions $\boldsymbol{u}, p$ and $\rho$ satisfying the corresponding integral identities.

LEMMA 2.1 For given $h>0$ and $\delta>0$ the problem (2.1)-(2.3) has a weak solution.

Proof. To solve the problem (2.1)-(2.3) we set

$$
\mathfrak{N}=\left\{\sigma \in L_{2}\left(\Omega_{T}\right): 0 \leqslant \sigma(\boldsymbol{x}, t) \leqslant \rho_{0}^{+} \text {a.e. in } \Omega_{T}\right\},
$$

and consider in $\Omega_{T}$ an initial boundary value problem for the linear system

$$
\nabla \cdot\left(\mathbb{P}^{(\varepsilon)}(\boldsymbol{u})\right)-\nabla p+(\sigma)_{(\delta)} \boldsymbol{F}=0, \quad \nabla \cdot \boldsymbol{u}=0,
$$

with the homogeneous boundary and initial conditions (2.3) for the function $\boldsymbol{u}$. 
The solvability of this problem is standard and follows from the estimates

$$
\begin{gathered}
\max _{0<t<T}\left\|\chi^{\varepsilon} \mathbb{D}(x, \boldsymbol{u})\right\|_{2, \Omega}(t)+\left\|\left(1-\chi^{\varepsilon}\right) \mathbb{D}(x, \boldsymbol{u})\right\|_{2, \Omega_{T}}+\|p\|_{2, \Omega_{T}} \leqslant C, \\
\max _{0<t<T}\left\|\chi^{\varepsilon} \mathbb{D}\left(x, \frac{\partial \boldsymbol{u}}{\partial t}\right)\right\|_{2, \Omega}(t)+\left\|\left(1-\chi^{\varepsilon}\right) \mathbb{D}\left(x, \frac{\partial \boldsymbol{u}}{\partial t}\right)\right\|_{2, \Omega_{T}} \leqslant \frac{C}{\delta},
\end{gathered}
$$

where $C=C\left(\rho_{0}^{+}, F, T\right)$.

To obtain the first estimate we multiply equation (2.4) by $\boldsymbol{u}$ and integrate by parts over $\Omega$ using the Hölder, Korn, Friedrichs-Poincaré and Gronwall inequalities (for more details see [8]). To get the second estimate we differentiate equation (2.4) with respect to time, multiply the result by $\partial \boldsymbol{u} / \partial t$ and integrate by parts over $\Omega$, repeating the same procedure as above.

Therefore, $\boldsymbol{u}$ and $\boldsymbol{v}=\mathbb{E}_{\Omega_{f}^{\varepsilon}}(\partial \boldsymbol{u} / \partial t)$ are continuous linear operators of $\sigma: \boldsymbol{u}=\Phi_{0}(\sigma), \boldsymbol{v}=$ $\Phi(\sigma)$. In particular,

$$
\max _{0<t<T}\left(\|\boldsymbol{v}\|_{2, \Omega}(t)+\|\nabla \boldsymbol{v}\|_{2, \Omega}(t)\right) \leqslant \frac{C}{\delta} F\|\sigma\|_{2, \Omega_{T}} .
$$

In (2.7) we have used the Korn inequality and estimates (2.6) and (1.1).

Next we consider in $\Omega_{T}$ a linear problem for the parabolic equation (2.2) with conditions (2.3) for the function $\rho$ with $\boldsymbol{v}=\mathbb{E}_{\Omega_{f}^{\varepsilon}}(\partial \boldsymbol{u} / \partial t)$ and $\boldsymbol{u}=\Phi(\sigma)$. As before, the solvability of this problem is a simple consequence of the maximum principle

$$
0 \leqslant \rho(\boldsymbol{x}, t) \leqslant \rho_{0}^{+} \quad \text { a.e. in } \Omega_{T},
$$

the energy equality

$$
\int_{\Omega}|\rho(\boldsymbol{x}, t)|^{2} \mathrm{~d} x+2 h \int_{0}^{t} \int_{\Omega}|\nabla \rho(\boldsymbol{x}, \tau)|^{2} \mathrm{~d} x \mathrm{~d} \tau=\int_{\Omega}\left|\rho_{h}^{(0)}(\boldsymbol{x})\right|^{2} \mathrm{~d} x
$$

and the estimate

$$
\max _{0<t<T} \int_{\Omega}|\rho(\boldsymbol{x}, t)|^{2} \mathrm{~d} x+2 h \int_{0}^{T} \int_{\Omega}|\nabla \rho(\boldsymbol{x}, t)|^{2} \mathrm{~d} x \mathrm{~d} t \leqslant\left|\rho_{0}^{+}\right|^{2}
$$

(for more details see [6]).

For fixed $h$ the problem (2.2), (2.3) defines a continuous operator $\rho=\Psi(v)$. In fact, if $\rho_{1}=$ $\Psi\left(\boldsymbol{v}_{1}\right)$ and $\rho_{2}=\Psi\left(\boldsymbol{v}_{2}\right)$, then for $\tilde{\rho}=\rho_{1}-\rho_{2}$ and $\tilde{\boldsymbol{v}}=\boldsymbol{v}_{1}-\boldsymbol{v}_{2}$ one has

$$
\begin{gathered}
\frac{1}{2} \frac{\mathrm{d}}{\mathrm{d} t} \int_{\Omega}|\tilde{\rho}(\boldsymbol{x}, t)|^{2} \mathrm{~d} x+h \int_{\Omega}|\nabla \tilde{\rho}|^{2} \mathrm{~d} x=J, \\
J=-\int_{\Omega} \tilde{\rho} \nabla \rho_{2} \cdot \tilde{\boldsymbol{v}} \mathrm{d} x=\int_{\Omega} \rho_{2} \nabla \tilde{\rho} \cdot \tilde{\boldsymbol{v}} \mathrm{d} x, \\
|J| \leqslant \frac{h}{2} \int_{\Omega}|\nabla \tilde{\rho}(\boldsymbol{x}, t)|^{2} \mathrm{~d} x+\frac{1}{2 h}\left|\rho_{0}^{+}\right|^{2} \int_{\Omega}|\tilde{\boldsymbol{v}}|^{2} \mathrm{~d} x .
\end{gathered}
$$

Thus,

$$
\left\|\Psi\left(\boldsymbol{v}_{1}\right)-\Psi\left(\boldsymbol{v}_{2}\right)\right\|_{2, \Omega_{T}} \leqslant \frac{\rho_{0}^{+}}{\sqrt{h}}\left\|\boldsymbol{v}_{1}-\boldsymbol{v}_{2}\right\|_{2, \Omega_{T}} .
$$


Let now $\rho=\Lambda(\sigma) \equiv \Psi(\Phi(\sigma))$. The operator $\Lambda$ is continuous in $\mathfrak{N}$ due to estimates (2.7) and (2.10), and transforms $\mathfrak{N}$ into itself by (2.8). The set $\mathfrak{N}$ is obviously convex and closed in $L_{2}\left(\Omega_{T}\right)$.

Moreover, due to well-known properties of the solutions to the linear parabolic equation (2.2) and embedding theorems [6], and estimate (2.7),

$$
\boldsymbol{v} \in L_{6}\left(\Omega_{T}\right), \quad \rho \in W_{6}^{2,1}\left(\Omega_{T}\right) \subset H^{\beta, \beta / 2}\left(\bar{\Omega}_{T}\right) .
$$

This last smoothness property of the function $\rho$ means that the operator $\Lambda$ is completely continuous. Applying the Schauder fixed point theorem [4] we get a fixed point $\rho_{(\delta, h)}$ of the operator $\Lambda$, which defines a solution $\left\{\rho_{(\delta, h)}, \boldsymbol{u}_{(\delta, h)}, p_{(\delta, h)}\right\}$ to the problem (2.1)-(2.3), satisfying estimates (2.5)-(2.9).

Now we derive the basic a priori estimates, which permit us to pass to the limit in (2.1)-(2.3) as $\delta \rightarrow 0$ and $h \rightarrow 0$.

LemMA 2.2 The solutions $\left\{\rho_{(\delta, h)}, \boldsymbol{u}_{(\delta, h)}, p_{(\delta, h)}\right\}$ to the problem (2.1)-(2.3) satisfy estimates (2.8), (2.9) for the density $\rho_{(\delta, h)}$, estimate $(2.5)$, and estimates

$$
\max _{0<t<T} \int_{\Omega}\left|\nabla \boldsymbol{v}_{(\delta, h)}(\boldsymbol{x}, t)\right|^{2} \mathrm{~d} x+\int_{0}^{T} \int_{\Omega}\left(\left|p_{(\delta, h)}\right|^{2}+\left|\nabla \frac{\partial \boldsymbol{u}_{(\delta, h)}}{\partial t}\right|^{2}\right) \mathrm{d} x \mathrm{~d} t \leqslant C
$$

for the pressure $p_{(\delta, h)}$, the liquid velocity $\boldsymbol{v}_{(\delta, h)}$ and the displacements $\boldsymbol{u}_{(\delta, h)}$, where the constant $C=C\left(\rho_{0}^{+}, F, T\right)$ is independent of $\varepsilon, \delta$ and $h$.

Proof. For the moment we omit the indices $h$ and $\delta$. As noted above, the approximation of $\rho$ by $(\rho)_{(\delta)}$ yields additional smoothness of the solution $\boldsymbol{u}$ to the problem (2.1)-(2.3) with respect to time. Now we prove that this additional smoothness does not depend on the small parameters $\varepsilon, \delta$ and $h$.

First, we multiply equation (2.1) by $\partial \boldsymbol{u} / \partial t$, integrate by parts over $\Omega$, transfer the time derivative in

$$
\int_{\Omega}(\rho)_{(\delta)} \boldsymbol{F} \cdot \frac{\partial \boldsymbol{u}}{\partial t} \mathrm{~d} x
$$

from $\boldsymbol{u}$ to $(\rho)_{(\delta)} \boldsymbol{F}$ and express the time derivative

$$
\frac{\partial(\rho)_{(\delta)}}{\partial t}= \begin{cases}\frac{1}{\delta} \int_{t-\delta}^{t} \frac{\partial \rho}{\partial \tau}(\boldsymbol{x}, \tau) \mathrm{d} \tau & \text { for } t>\delta, \\ \frac{1}{\delta} \int_{0}^{t} \frac{\partial \rho}{\partial \tau}(\boldsymbol{x}, \tau) \mathrm{d} \tau & \text { for } t<\delta,\end{cases}
$$

using equation (2.2) in the form

$$
\frac{\partial \rho}{\partial t}=\nabla \cdot(h \nabla \rho-\rho \boldsymbol{v}) .
$$

We have

$$
\alpha_{\mu} \int_{\Omega} \chi^{\varepsilon}\left|\mathbb{D}\left(x, \frac{\partial \boldsymbol{u}}{\partial t}\right)\right|^{2} \mathrm{~d} x+\frac{\alpha_{\lambda}}{2} \frac{\mathrm{d}}{\mathrm{d} t} \int_{\Omega}\left(1-\chi^{\varepsilon}\right)|\mathbb{D}(x, \boldsymbol{u})|^{2} \mathrm{~d} x=I_{0}(t),
$$

where

$$
-I_{0}(t)=I_{0,0}(t)+\int_{\Omega}(\rho)_{(\delta)}\left(\frac{\partial \boldsymbol{F}}{\partial t} \cdot \boldsymbol{u}\right) \mathrm{d} x
$$


with

$$
I_{0,0}(t)= \begin{cases}\int_{\Omega}\left(\frac{1}{\delta} \int_{t-\delta}^{t}(h \nabla \rho-\rho \boldsymbol{v})(\boldsymbol{x}, \tau) \mathrm{d} \tau\right) \cdot \nabla(\boldsymbol{F} \cdot \boldsymbol{u})(\boldsymbol{x}, t) \mathrm{d} x & \text { for } t>\delta, \\ \int_{\Omega}\left(\frac{1}{\delta} \int_{0}^{t}(h \nabla \rho-\rho \boldsymbol{v})(\boldsymbol{x}, \tau) \mathrm{d} \tau\right) \cdot \nabla(\boldsymbol{F} \cdot \boldsymbol{u})(\boldsymbol{x}, t) \mathrm{d} x & \text { for } t<\delta .\end{cases}
$$

Everything that we have done to get (2.12) is just a formal procedure, but we may do it rigorously using the corresponding integral identities.

It is easy to see that for any positive $\gamma$,

$$
\begin{aligned}
\int_{0}^{t}\left|I_{0}(\tau)\right| \mathrm{d} \tau \leqslant & \gamma \int_{0}^{t} \int_{\Omega}|\boldsymbol{v}|^{2} \mathrm{~d} x \mathrm{~d} \tau+\left(\frac{1}{4 \gamma}+\frac{1}{2}\right)\left(\rho_{0}^{+} F\right)^{2} \int_{0}^{t} \int_{\Omega}\left(|\nabla \boldsymbol{u}|^{2}+|\boldsymbol{u}|^{2}\right) \mathrm{d} x \mathrm{~d} \tau \\
& +\frac{h}{2} \int_{0}^{t} \int_{\Omega} h|\nabla \rho|^{2} \mathrm{~d} x \mathrm{~d} \tau+2 \rho_{0}^{+} F\left(\int_{0}^{t} \int_{\Omega}|\boldsymbol{u}|^{2} \mathrm{~d} x \mathrm{~d} \tau\right)^{1 / 2} .
\end{aligned}
$$

After integrating (2.12) with respect to time and taking into account estimates (2.5), (2.8) and assumption (0.13) one has

$$
\begin{aligned}
\int_{0}^{t} \int_{\Omega} \chi^{\varepsilon}\left|\mathbb{D}\left(x, \frac{\partial \boldsymbol{u}}{\partial t}(\boldsymbol{x}, \tau)\right)\right|^{2} \mathrm{~d} x \mathrm{~d} \tau+\int_{\Omega}|\mathbb{D}(x, \boldsymbol{u}(\boldsymbol{x}, t))|^{2} \mathrm{~d} x \\
\quad \leqslant C \gamma \int_{0}^{t} \int_{\Omega}|\boldsymbol{v}(\boldsymbol{x}, \tau)|^{2} \mathrm{~d} x \mathrm{~d} \tau+C\left(\frac{1}{\gamma}+1\right)\left(\rho_{0}^{+} F+1\right)^{4}+C\left(\rho_{0}^{+}\right)^{2} .
\end{aligned}
$$

Choosing $C \gamma \leqslant 1 / 2$ we get

$$
\int_{0}^{T} \int_{\Omega} \chi^{\varepsilon}\left|\mathbb{D}\left(x, \frac{\partial \boldsymbol{u}}{\partial t}(\boldsymbol{x}, t)\right)\right|^{2} \mathrm{~d} x \mathrm{~d} t \leqslant C\left(\rho_{0}^{+} F+1\right)^{4},
$$

where $C$ is independent of $\varepsilon, \delta$ and $h$.

This last estimate, estimate (1.1), and the Korn inequality imply

$$
\int_{0}^{T} \int_{\Omega}|\nabla \boldsymbol{v}(\boldsymbol{x}, t)|^{2} \mathrm{~d} x \mathrm{~d} t \leqslant C\left(\rho_{0}^{+} F+1\right)^{4} .
$$

Since $\boldsymbol{v} \in L_{2}\left((0, T) ; \stackrel{\circ}{W}_{2}^{1}(\Omega)\right)$ we may use the Friedrichs-Poincaré inequality

$$
\int_{0}^{T} \int_{\Omega}|\boldsymbol{v}(\boldsymbol{x}, t)|^{2} \mathrm{~d} x \mathrm{~d} t \leqslant C \int_{0}^{T} \int_{\Omega}|\nabla \boldsymbol{v}(\boldsymbol{x}, t)|^{2} \mathrm{~d} x \mathrm{~d} t
$$

with a constant $C$ independent of $\varepsilon$.

Estimates (2.14) and (2.15) yield

$$
\int_{0}^{T} \int_{\Omega}|\boldsymbol{v}(\boldsymbol{x}, t)|^{2} \mathrm{~d} x \mathrm{~d} t \leqslant C\left(\rho_{0}^{+} F+1\right)^{4}
$$


Now, we repeat the same procedure for time derivatives. Namely, we differentiate the equation (2.1) with respect to time, multiply the result by $\partial \boldsymbol{u} / \partial t$, and integrate over $\Omega$ :

$$
\begin{gathered}
\frac{\alpha_{\mu}}{2} \frac{\mathrm{d}}{\mathrm{d} t} \int_{\Omega} \chi^{\varepsilon}\left|\mathbb{D}\left(x, \frac{\partial \boldsymbol{u}}{\partial t}\right)\right|^{2} \mathrm{~d} x+\alpha_{\lambda} \int_{\Omega}\left(1-\chi^{\varepsilon}\right)\left|\mathbb{D}\left(x, \frac{\partial \boldsymbol{u}}{\partial t}\right)\right|^{2} \mathrm{~d} x=I_{1}(t), \\
-I_{1}(t)=I_{1,0}(t)+\int_{\Omega}(\rho)(\delta)\left(\frac{\partial \boldsymbol{F}}{\partial t} \cdot \frac{\partial \boldsymbol{u}}{\partial t}\right) \mathrm{d} x, \\
I_{1,0}(t)=\left\{\begin{array}{l}
\int_{\Omega}\left(\frac{1}{\delta} \int_{t-\delta}^{t}(h \nabla \rho-\rho \boldsymbol{v})(\boldsymbol{x}, \tau) \mathrm{d} \tau\right) \cdot \nabla\left(\boldsymbol{F} \cdot \frac{\partial \boldsymbol{u}}{\partial t}\right)(\boldsymbol{x}, t) \mathrm{d} x \quad \text { for } t>\delta, \\
\int_{\Omega}\left(\frac{1}{\delta} \int_{0}^{t}(h \nabla \rho-\rho \boldsymbol{v})(\boldsymbol{x}, \tau) \mathrm{d} \tau\right) \cdot \nabla\left(\boldsymbol{F} \cdot \frac{\partial \boldsymbol{u}}{\partial t}\right)(\boldsymbol{x}, t) \mathrm{d} x \quad \text { for } t<\delta,
\end{array}\right. \\
\int_{0}^{t}\left|I_{1}(\tau)\right| \mathrm{d} \tau \leqslant(\gamma+h) \int_{0}^{t} \int_{\Omega}\left(\left|\nabla\left(\frac{\partial \boldsymbol{u}}{\partial t}\right)\right|^{2}+\left|\frac{\partial \boldsymbol{u}}{\partial t}\right|^{2}\right) \mathrm{d} x \mathrm{~d} \tau \\
+\left(\rho_{0}^{+} \cdot F\right)^{2}\left(\int_{0}^{t} \int_{\Omega}\left(\frac{1}{4 \gamma}|\boldsymbol{v}|^{2}+h|\nabla \rho|^{2}\right) \mathrm{d} x \mathrm{~d} \tau+1\right) .
\end{gathered}
$$

Integrating (2.17) with respect to time and using the Friedrichs-Poincaré inequality

$$
\int_{\Omega}\left|\frac{\partial \boldsymbol{u}}{\partial t}\right|^{2} \mathrm{~d} x \leqslant C \int_{\Omega}\left|\nabla \frac{\partial \boldsymbol{u}}{\partial t}\right|^{2} \mathrm{~d} x
$$

the Korn inequality

$$
\int_{\Omega}\left|\nabla \frac{\partial \boldsymbol{u}}{\partial t}\right|^{2} \mathrm{~d} x \leqslant C \int_{\Omega}\left|\mathbb{D}\left(x, \frac{\partial \boldsymbol{u}}{\partial t}\right)\right|^{2} \mathrm{~d} x
$$

the evident decomposition

$$
\int_{\Omega}\left|\mathbb{D}\left(x, \frac{\partial \boldsymbol{u}}{\partial t}\right)\right|^{2} \mathrm{~d} x=\int_{\Omega} \chi^{\varepsilon}\left|\mathbb{D}\left(x, \frac{\partial \boldsymbol{u}}{\partial t}\right)\right|^{2} \mathrm{~d} x+\int_{\Omega}\left(1-\chi^{\varepsilon}\right)\left|\mathbb{D}\left(x, \frac{\partial \boldsymbol{u}}{\partial t}\right)\right|^{2} \mathrm{~d} x,
$$

and estimates (2.9) and (2.16) we obtain

$$
\begin{aligned}
& \frac{\alpha_{\mu}}{2} \int_{\Omega} \chi^{\varepsilon}\left|\mathbb{D}\left(x, \frac{\partial \boldsymbol{u}}{\partial t}(\boldsymbol{x}, t)\right)\right|^{2} \mathrm{~d} x+\alpha_{\lambda} \int_{0}^{t} \int_{\Omega}\left(1-\chi^{\varepsilon}\right)\left|\mathbb{D}\left(x, \frac{\partial \boldsymbol{u}}{\partial \tau}(\boldsymbol{x}, \tau)\right)\right|^{2} \mathrm{~d} x \mathrm{~d} \tau \\
& \quad \leqslant(\gamma+h) C\left(\int_{\Omega} \chi^{\varepsilon}\left|\mathbb{D}\left(x, \frac{\partial \boldsymbol{u}}{\partial t}\right)\right|^{2} \mathrm{~d} x+\int_{\Omega}\left(1-\chi^{\varepsilon}\right)\left|\mathbb{D}\left(x, \frac{\partial \boldsymbol{u}}{\partial t}\right)\right|^{2} \mathrm{~d} x\right)+C\left(\rho_{0}^{+} \cdot F\right)^{6} .
\end{aligned}
$$

Therefore

$$
\max _{0<t<T} \int_{\Omega} \chi^{\varepsilon}\left|\mathbb{D}\left(x, \frac{\partial \boldsymbol{u}}{\partial t}(\boldsymbol{x}, t)\right)\right|^{2} \mathrm{~d} x+\int_{0}^{T} \int_{\Omega}\left|\nabla \frac{\partial \boldsymbol{u}}{\partial t}\right|^{2} \mathrm{~d} x \mathrm{~d} t \leqslant C\left(\rho_{0}^{+} F+1\right)^{6} .
$$

The last estimate, (1.1) and the Korn inequality imply

$$
\max _{0<t<T} \int_{\Omega}|\nabla \boldsymbol{v}(\boldsymbol{x}, t)|^{2} \mathrm{~d} x \leqslant C\left(\rho_{0}^{+} F+1\right)^{6} .
$$


Estimate (2.11) for the pressure $p$ follows from equation (2.1) as an estimate for the bounded linear functional on $L_{2}\left((0, T) ; \stackrel{\circ}{2}_{2}^{1}(\Omega)\right)$ in the form

$$
\int_{\Omega_{T}} p \nabla \cdot \boldsymbol{\varphi} \mathrm{d} x \mathrm{~d} t=\int_{\Omega_{T}}\left(\mathbb{P}^{(\varepsilon)}(\boldsymbol{u}): \mathbb{D}(x, \boldsymbol{\varphi})+(\rho)_{(\delta)} \boldsymbol{F} \cdot \boldsymbol{\varphi}\right) \mathrm{d} x \mathrm{~d} t,
$$

and estimates (2.8) and (2.18).

As a last step we pass to the limit as $\delta \rightarrow 0$. We do it in (2.20) and in the integral identity

$$
\int_{\Omega_{T}}\left(\rho\left(\frac{\partial \psi}{\partial t}+\boldsymbol{v} \cdot \nabla \psi\right)-h \nabla \rho \cdot \nabla \psi\right) \mathrm{d} x \mathrm{~d} t=-\int_{\Omega} \rho_{h}^{(0)}(\boldsymbol{x}) \psi(\boldsymbol{x}, 0) \mathrm{d} x,
$$

with arbitrary smooth functions $\varphi$ and $\psi(\varphi$, and $\psi$ vanish at $S$, and $\psi$ vanishes at $t=T)$.

Estimates (2.8), (2.9), (2.11) guarantee the inclusion

$$
\frac{\partial \rho_{(\delta, h)}}{\partial t} \in L_{2}\left((0, T) ; W_{2}^{-1}(\Omega)\right)
$$

and the norms of all these derivatives in $L_{2}\left((0, T) ; W_{2}^{-1}(\Omega)\right)$ are uniformly bounded with respect to $\delta, h$ and $\varepsilon$.

From these estimates, the above mentioned inclusion, and well-known compactness results [7], we may choose some subsequence from $\{\delta>0\}$ such that the sequences

$$
\left\{p_{(\delta, h)}\right\},\left\{\nabla \boldsymbol{u}_{(\delta, h)}\right\},\left\{\nabla \frac{\partial \boldsymbol{u}_{(\delta, h)}}{\partial t}\right\},\left\{\boldsymbol{v}_{(\delta, h)}\right\} \text { and }\left\{\nabla \rho_{(\delta, h)}\right\}
$$

converge weakly in $L_{2}\left(\Omega_{T}\right)$ as $\delta \rightarrow 0$ to functions

$$
p_{(h)}, \nabla \boldsymbol{u}_{(h)}, \nabla \frac{\partial \boldsymbol{u}_{(h)}}{\partial t}, \boldsymbol{v}_{(h)}=\mathbb{E}_{\Omega_{f}^{\varepsilon}}\left(\frac{\partial \boldsymbol{u}_{(h)}}{\partial t}\right) \text { and } \nabla \rho_{(h)}
$$

respectively, and the sequence $\left\{\rho_{(\delta, h)}\right\}$ converges strongly in $L_{2}\left(\Omega_{T}\right)$ as $\delta \rightarrow 0$ to a function $\rho_{(h)}$. Letting $\delta \rightarrow 0$ in the integral identities (2.20) and (2.21) we arrive at a weak solution $\left\{\boldsymbol{u}_{(h)}, p_{(h)}, \rho_{(h)}\right\}$ to the approximate problem

$$
\begin{gathered}
\nabla \cdot\left(\mathbb{P}^{(\varepsilon)}\left(\boldsymbol{u}_{(h)}\right)\right)-\nabla p_{(h)}+\rho_{(h)} \boldsymbol{F}=0, \quad \nabla \cdot \boldsymbol{u}_{(h)}=0, \\
\frac{\partial \rho_{(h)}}{\partial t}+\boldsymbol{v}_{(h)} \cdot \nabla \rho_{(h)}=h \Delta \rho_{(h)},
\end{gathered}
$$

satisfying the initial and boundary conditions (2.3).

Finally, to prove Theorem 1 we have to pass to the limit as $h \rightarrow 0$ in (2.3), (2.22), (2.23).

To do that we derive the main a priori estimate.

Lemma 2.3 The solutions $\left\{\rho_{(h)}, \boldsymbol{u}_{(h)}, p_{(h)}\right\}$ to the problem (2.22), (2.23) and (2.3) satisfy estimates (2.8), (2.9), (2.11) and the estimate

$$
\int_{\Omega}\left|\nabla \boldsymbol{v}_{(h)}\left(\boldsymbol{x}, t_{1}\right)-\nabla \boldsymbol{v}_{(h)}\left(\boldsymbol{x}, t_{2}\right)\right|^{2} \mathrm{~d} x \leqslant C\left|t_{1}-t_{2}\right|^{1 / 2},
$$

with a constant $C$ independent of $h$ and $\varepsilon$. 
Proof. As before, we omit the index $h$ for the moment. It is clear that we only have to prove (2.24). In the same way as in Lemma 2.2 we get the integral identity

$$
\alpha_{\mu} \frac{\mathrm{d}}{\mathrm{d} t} \int_{\Omega} \chi^{\varepsilon} \mathbb{D}\left(x, \frac{\partial \boldsymbol{u}}{\partial t}(\boldsymbol{x}, t)\right): \mathbb{D}(x, \varphi(\boldsymbol{x})) \mathrm{d} x=I_{2}(t),
$$

where

$$
\begin{aligned}
I_{2}(t)= & -\alpha_{\lambda} \int_{\Omega}\left(1-\chi^{\varepsilon}\right) \mathbb{D}\left(x, \frac{\partial \boldsymbol{u}}{\partial t}(\boldsymbol{x}, t)\right): \mathbb{D}(x, \varphi(\boldsymbol{x})) \mathrm{d} x \\
& +\int_{\Omega}(h \nabla \rho-\rho \boldsymbol{v})(\boldsymbol{x}, t) \cdot \nabla(\boldsymbol{F}(\boldsymbol{x}, t) \cdot \boldsymbol{\varphi}(\boldsymbol{x})) \mathrm{d} x+\int_{\Omega} \rho(\boldsymbol{x}, t)\left(\frac{\partial \boldsymbol{F}}{\partial t}(\boldsymbol{x}, t) \cdot \boldsymbol{\varphi}(\boldsymbol{x})\right) \mathrm{d} x,
\end{aligned}
$$

which holds true for any solenoidal function $\varphi \in \stackrel{\circ}{W}_{2}^{1}(\Omega)$.

Due to estimates (2.8), (2.9), (2.11), $I_{2} \in L_{2}(0, T)$ and

$$
\int_{t_{1}}^{t_{2}}\left|I_{2}(t)\right| \mathrm{d} t \leqslant C \int_{t_{1}}^{t_{2}}\left\|h|\nabla \rho|+|\boldsymbol{v}|+\left|\nabla \frac{\partial \boldsymbol{u}}{\partial t}\right|+1\right\|_{2, \Omega}(t) \mathrm{d} t\|\nabla \varphi\|_{2, \Omega} \leqslant C\left|t_{1}-t_{2}\right|^{1 / 2}\|\nabla \varphi\|_{2, \Omega} .
$$

Therefore,

$$
\int_{\Omega} \chi^{\varepsilon} \mathbb{D}\left(x, \frac{\partial \tilde{\boldsymbol{u}}}{\partial t}\right): \mathbb{D}(x, \varphi) \mathrm{d} x \leqslant C\left|t_{1}-t_{2}\right|^{1 / 2}\|\nabla \varphi\|_{2, \Omega}
$$

where

$$
\frac{\partial \tilde{\boldsymbol{u}}}{\partial t}=\frac{\partial \boldsymbol{u}}{\partial t}\left(\boldsymbol{x}, t_{2}\right)-\frac{\partial \boldsymbol{u}}{\partial t}\left(\boldsymbol{x}, t_{1}\right) .
$$

In particular, for $\varphi=\tilde{\boldsymbol{v}}=\boldsymbol{v}\left(\boldsymbol{x}, t_{2}\right)-\boldsymbol{v}\left(\boldsymbol{x}, t_{1}\right)$,

$$
\|\nabla \varphi\|_{2, \Omega} \leqslant C, \quad \forall t_{1}, t_{2} \in(0, T),
$$

and

$$
\int_{\Omega} \chi^{\varepsilon} \mathbb{D}\left(x, \frac{\partial \tilde{\boldsymbol{u}}}{\partial t}\right): \mathbb{D}(x, \tilde{\boldsymbol{v}}) \mathrm{d} x \leqslant C\left|t_{1}-t_{2}\right|^{1 / 2} .
$$

But, by the definition of the extension $v$,

$$
\chi^{\varepsilon} \mathbb{D}(x, \tilde{\boldsymbol{v}})=\chi^{\varepsilon} \mathbb{D}\left(x, \frac{\partial \tilde{\boldsymbol{u}}}{\partial t}\right) .
$$

Thus,

$$
\int_{\Omega} \chi^{\varepsilon} \mathbb{D}\left(x, \frac{\partial \tilde{\boldsymbol{u}}}{\partial t}\right): \mathbb{D}\left(x, \frac{\partial \tilde{\boldsymbol{u}}}{\partial t}\right) \mathrm{d} x=\int_{\Omega} \chi^{\varepsilon} \mathbb{D}\left(x, \frac{\partial \tilde{\boldsymbol{u}}}{\partial t}\right): \mathbb{D}(x, \tilde{\boldsymbol{v}}) \mathrm{d} x \leqslant C\left|t_{1}-t_{2}\right|^{1 / 2}
$$

The statement of the lemma now follows from the last estimate, (1.1) and the Korn inequality.

Lemmas 2.2 and 2.3 permit us to find a subsequence from $\{h>0\}$ such that the sequences

$$
\left\{p_{(h)}\right\},\left\{\nabla \boldsymbol{u}_{(h)}\right\},\left\{\chi^{\varepsilon} \nabla \frac{\partial \boldsymbol{u}_{(h)}}{\partial t}\right\},\left\{\boldsymbol{v}_{(h)}\right\},\left\{\nabla \boldsymbol{v}_{(h)}\right\} \text { and }\left\{\rho_{(h)}\right\}
$$


converge weakly in $L_{2}\left(\Omega_{T}\right)$ as $h \rightarrow 0$ to the functions

$$
p^{\varepsilon}, \nabla \boldsymbol{u}^{\varepsilon}, \chi^{\varepsilon} \nabla \frac{\partial \boldsymbol{u}^{\varepsilon}}{\partial t}, \boldsymbol{v}^{\varepsilon}, \nabla \boldsymbol{v}^{\varepsilon} \text { and } \rho^{\varepsilon}
$$

respectively [7], and the sequence $\left\{\boldsymbol{v}_{(h)}\right\}$ converges strongly in $L_{2}\left(\Omega_{T}\right)$ as $h \rightarrow 0$ to the function $\boldsymbol{v}^{\varepsilon}=\mathbb{E}_{\Omega_{f}^{\varepsilon}}\left(\partial \boldsymbol{u}^{\varepsilon} / \partial t\right)$.

In fact, to prove the last statement we fix a countable dense set $\left(t_{(k)}\right)_{k=1}^{\infty}$ in $(0, T)$ and choose a subsequence from $\{h>0\}$ such that the sequences $\left\{\nabla \boldsymbol{v}_{(h)}\left(\boldsymbol{x}, t_{(k)}\right)\right\}$ converge weakly in $L_{2}(\Omega)$ as $h \rightarrow 0$ for all $k=1,2, \ldots$. This is possible due to estimate (2.11) and the standard diagonal procedure. The last fact and estimate (2.24) guarantee the weak convergence in $L_{2}(\Omega)$ of the sequences $\left\{\nabla \boldsymbol{v}_{(h)}(\boldsymbol{x}, t)\right\}$ for all $t \in(0, T)$. Now we apply the completely continuous imbedding of $\stackrel{\circ}{W}_{2}^{1}(\Omega)$ into $L_{2}(\Omega)$ (see [7]) and conclude that the sequence $\left\{\boldsymbol{v}_{(h)}(\boldsymbol{x}, t)\right\}$ converges strongly in $L_{2}(\Omega)$ for all $t \in(0, T)$. The limiting procedure in the integral identities (2.20) and (2.21) as $h \rightarrow 0$ proves the statement of Theorem 1 .

\section{Proof of Theorem 2}

To prove the theorem we just have to pass to the limit as $\varepsilon \rightarrow 0$. One already knows how to pass to the limit in the convection term $\rho^{\varepsilon} \cdot \boldsymbol{v}^{\varepsilon}$. But for $\varepsilon$ variable additional nonlinear terms appear, containing the characteristic function $\chi^{\varepsilon}$ of the pore space. Generally the sequence $\left\{\chi^{\varepsilon}\right\}$ converges only weakly in $L_{2}\left(\Omega_{T}\right)$. Therefore we cannot directly pass to the limit in the integral identity (1.5), because the bounded sequence $\left\{\mathbb{D}\left(x, \boldsymbol{w}^{\varepsilon}\right)\right\}$ may converge only weakly in $L_{2}\left(\Omega_{T}\right)$. This is the main problem in homogenization theory and we will use the two-scale convergence method, suggested by G. Nguetseng [10]. For this method all functions $\varphi$ of the form $\varphi=\varphi(x, x / \varepsilon, t), 1$-periodic in the variable $\boldsymbol{y}=\boldsymbol{x} / \varepsilon$, are already included in the class of test functions.

More precisely, the sequence $\left\{u^{\varepsilon}(\boldsymbol{x}, t)\right\}$ is said to be two-scale convergent in $L_{2}\left(\Omega_{T}\right)$ if there exists a function $U \in L_{2}\left(\Omega_{T} \times Y\right)$ 1-periodic in $\boldsymbol{y} \in Y$ such that

$$
\int_{\Omega_{T}} u^{\varepsilon} \varphi(\boldsymbol{x}, \boldsymbol{x} / \varepsilon, t) \mathrm{d} x \mathrm{~d} t \rightarrow \int_{Y} \int_{\Omega_{T}} U(\boldsymbol{x}, \boldsymbol{y}, t) \varphi(\boldsymbol{x}, \boldsymbol{y}, t) \mathrm{d} x \mathrm{~d} t \mathrm{~d} y
$$

for any smooth function $\varphi(\boldsymbol{x}, \boldsymbol{y}, t)$ 1-periodic in $\boldsymbol{y} \in Y$, in particular, for $\varphi(\boldsymbol{x}, \boldsymbol{y}, t)=\varphi_{0}(\boldsymbol{y})$. $h(\boldsymbol{x}, t)$, where $\varphi_{0} \in L_{2}(Y)$ and $h \in L_{\infty}\left(\Omega_{T}\right)$.

Note that if $\left\{u^{\varepsilon}(\boldsymbol{x}, t)\right\}$ two-scale converges in $L_{2}\left(\Omega_{T}\right)$ to a function $U(\boldsymbol{x}, \boldsymbol{y}, t)$, then it converges weakly in $L_{2}\left(\Omega_{T}\right)$ to the function

$$
u(\boldsymbol{x}, t)=\langle U\rangle_{Y}=\int_{Y} U(\boldsymbol{x}, \boldsymbol{y}, t) \mathrm{d} y .
$$

The main result in [10] states that

1) Any sequence bounded in $L_{2}\left(\Omega_{T}\right)$ contains a two-scale convergent subsequence.

2) Any sequence $\left\{u^{\varepsilon}\right\}$ bounded in $L_{2}\left((0, T) ; W_{2}^{1}(\Omega)\right)$ contains a subsequence $\left\{u^{\varepsilon_{k}}\right\}$ such that $\left\{u^{\varepsilon_{k}}\right\}$ is two-scale convergent in $L_{2}\left(\Omega_{T}\right)$ to a function $u(\boldsymbol{x}, t) \in L_{2}\left((0, T) ; W_{2}^{1}(\Omega)\right)$ and $\left\{\nabla u^{\varepsilon_{k}}\right\}$ two-scale converges to $\nabla u(\boldsymbol{x}, t)+\nabla_{y} U(\boldsymbol{x}, \boldsymbol{y}, t)$, where $U(\boldsymbol{x}, \boldsymbol{y}, t)$ is 1-periodic in $\boldsymbol{y} \in Y$ and $U, \nabla_{y} U \in L_{2}\left(\Omega_{T} \times Y\right)$. 
Due to the properties of the domains $\Omega_{f}^{\varepsilon}$ and $\Omega_{s}^{\varepsilon}$ the function $\chi^{\varepsilon}$ has the form $\chi^{\varepsilon}=\chi(x / \varepsilon)$, where $\chi(\boldsymbol{y})$ is 1-periodic (it is a periodic extension of the characteristic function of the domain $Y_{f}$ in $Y$ ). Thus, the limit as $\varepsilon \rightarrow 0$ in the term $\chi^{\varepsilon} \mathbb{D}\left(x, \boldsymbol{w}^{\varepsilon}\right)$ becomes trivial.

As before, we divide the proof into several independent statements.

LEMMA 3.1 There exists a subsequence of small parameters $\varepsilon \searrow 0$ such that

(1) $\left\{\boldsymbol{w}^{\varepsilon}\right\}$ converges weakly in $L_{2}\left((0, T) ; \stackrel{\circ}{W}_{2}^{1}(\Omega)\right)$ to a function $\boldsymbol{w}$;

(2) $\left\{\rho^{\varepsilon}\right\}$ converges weakly in $L_{2}\left(\Omega_{T}\right)$ to a function $\rho$;

(3) $\left\{p^{\varepsilon}\right\}$ converges weakly in $L_{2}\left(\Omega_{T}\right)$ to a function $p$;

(4) there exist 1-periodic (in $y \in Y$ ) functions

$$
P(\boldsymbol{x}, t, \boldsymbol{y}), \boldsymbol{W}(\boldsymbol{x}, t, \boldsymbol{y}), \boldsymbol{V}(\boldsymbol{x}, t, \boldsymbol{y}), \quad P, \nabla \boldsymbol{W}, \nabla \boldsymbol{V} \in L_{2}\left(\Omega_{T} \times Y\right),
$$

such that the sequences $\left\{p^{\varepsilon}\right\},\left\{\nabla \boldsymbol{w}^{\varepsilon}\right\},\left\{\nabla\left(\partial \boldsymbol{w}^{\varepsilon} / \partial t\right)\right\}$ and $\left\{\nabla \boldsymbol{v}^{\varepsilon}\right\}$ two-scale converge to

$$
P, \nabla_{x} \boldsymbol{w}+\nabla_{y} \boldsymbol{W}, \nabla_{x}\left(\frac{\partial \boldsymbol{w}}{\partial t}\right)+\nabla_{y}\left(\frac{\partial \boldsymbol{W}}{\partial t}\right) \text { and } \nabla_{x}\left(\frac{\partial \boldsymbol{w}}{\partial t}\right)+\nabla_{y} \boldsymbol{V}
$$

respectively;

(5) $\left\{\partial \boldsymbol{w}^{\varepsilon} / \partial t\right\}$ converges weakly in $L_{2}\left((0, T) ; \stackrel{\circ}{W}_{2}^{1}(\Omega)\right)$ and strongly in $L_{2}\left(\Omega_{T}\right)$ to $\partial \boldsymbol{w} / \partial t$;

(6) $\left\{\boldsymbol{v}^{\varepsilon}\right\}$, where $\boldsymbol{v}^{\varepsilon}=\mathbb{E}_{\Omega_{f}^{\varepsilon}}\left(\partial \boldsymbol{w}^{\varepsilon} / \partial t\right)$, converges strongly in $L_{2}\left(\Omega_{T}\right)$ to $\partial \boldsymbol{w} / \partial t$.

Proof. The proof of the first three statements and the proof of the strong convergence of $\left\{\boldsymbol{v}^{\varepsilon}\right\}$ in $L_{2}\left(\Omega_{T}\right)$ to some limit $\boldsymbol{v}$ repeats similar proofs in the previous section.

(4) This statement follows from the results of G. Nguetseng [10] and estimates (1.6) and (1.7). We only have to prove that the weak limits of $\left\{\boldsymbol{v}^{\varepsilon}\right\}$ and $\left\{\partial \boldsymbol{w}^{\varepsilon} / \partial t\right\}$ coincide.

First of all note that if $\left\{\boldsymbol{v}^{\varepsilon}\right\}$ two-scale converges to the function $\boldsymbol{V}_{0}(\boldsymbol{x}, t, \boldsymbol{y})$ and $\left\{\nabla \boldsymbol{v}^{\varepsilon}\right\}$ two-scale converges to $\nabla_{x} \boldsymbol{v}(\boldsymbol{x}, t)+\nabla_{y} \boldsymbol{V}(\boldsymbol{x}, t, \boldsymbol{y})$, then $\boldsymbol{V}_{0}(\boldsymbol{x}, t, \boldsymbol{y})=\boldsymbol{v}(\boldsymbol{x}, t)$. Thus, the sequences $\left\{\boldsymbol{v}^{\varepsilon}\right\}$ and $\left\{\partial \boldsymbol{w}^{\varepsilon} / \partial t\right\}$ two-scale converge to $\boldsymbol{v}(\boldsymbol{x}, t)$ and $(\partial \boldsymbol{w} / \partial t)(\boldsymbol{x}, t)$ respectively. Taking now the two-scale limit in the equality

$$
\chi\left(\frac{x}{\varepsilon}\right)\left(\frac{\partial \boldsymbol{w}^{\varepsilon}}{\partial t}-\boldsymbol{v}^{\varepsilon}\right)=0
$$

we arrive at

$$
\chi(\boldsymbol{y})\left(\frac{\partial \boldsymbol{w}}{\partial t}(\boldsymbol{x}, t)-\boldsymbol{v}(\boldsymbol{x}, t)\right)=0 \quad \text { a.e. in } \Omega_{T} \times Y,
$$

which proves (4) and, at the same time, the first statement in (5). To prove the second part we use (6), (1.7) and the Friedrichs-Poincaré inequality [8]:

$$
\int_{\Omega}\left|\frac{\partial \boldsymbol{w}^{\varepsilon}}{\partial t}-\boldsymbol{v}^{\varepsilon}\right|^{2} \mathrm{~d} x=\int_{\Omega}\left(1-\chi^{\varepsilon}\right)\left|\frac{\partial \boldsymbol{w}^{\varepsilon}}{\partial t}-\boldsymbol{v}^{\varepsilon}\right|^{2} \mathrm{~d} x \leqslant \varepsilon^{2} C \int_{\Omega}\left(\left|\nabla \frac{\partial \boldsymbol{w}^{\varepsilon}}{\partial t}\right|^{2}+\left|\nabla \boldsymbol{v}^{\varepsilon}\right|^{2}\right) \mathrm{d} x .
$$

We have

$$
\begin{aligned}
\int_{\Omega_{T}}\left|\frac{\partial \boldsymbol{w}^{\varepsilon}}{\partial t}-\frac{\partial \boldsymbol{w}}{\partial t}\right|^{2} \mathrm{~d} x \mathrm{~d} t & =\int_{\Omega_{T}}\left|\frac{\partial \boldsymbol{w}^{\varepsilon}}{\partial t}-\boldsymbol{v}^{\varepsilon}\right|^{2} \mathrm{~d} x \mathrm{~d} t+\int_{\Omega_{T}}\left|\boldsymbol{v}^{\varepsilon}-\frac{\partial \boldsymbol{w}}{\partial t}\right|^{2} \mathrm{~d} x \mathrm{~d} t \\
& \leqslant \varepsilon^{2} C+\int_{\Omega}\left|\frac{\partial \boldsymbol{w}}{\partial t}-\boldsymbol{v}^{\varepsilon}\right|^{2} \mathrm{~d} x \mathrm{~d} t .
\end{aligned}
$$


LEMma 3.2 The limiting density $\rho$ satisfies the transport equation (1.12) in the form of the integral identity

$$
\int_{\Omega_{T}} \rho\left(\frac{\partial \xi}{\partial t}+\frac{\partial \boldsymbol{w}}{\partial t} \cdot \nabla \xi\right) \mathrm{d} x \mathrm{~d} t+\int_{\Omega} \rho^{(0)}(\boldsymbol{x}) \xi(\boldsymbol{x}, 0) \mathrm{d} x=0
$$

for an arbitrary smooth function $\xi$ with $\xi(x, T)=0$.

This statement becomes trivial due to the previous lemma.

The proofs of the following three lemmas may be found in [8].

LEMma 3.3 The limiting functions $\rho, p, \boldsymbol{w}$ and $\boldsymbol{W}$ satisfy in $\Omega_{T}$ the system of macroscopic equations

$$
\begin{gathered}
\nabla_{x} \cdot \mathbb{P}^{(0)}-\nabla p+\rho \boldsymbol{F}=0, \quad \mathbb{P}^{(0)}=\langle\widetilde{\mathbb{P}}\rangle_{Y}, \\
\widetilde{\mathbb{P}}=\mu_{0} \chi(\boldsymbol{y})\left(\mathbb{D}\left(x, \frac{\partial \boldsymbol{w}}{\partial t}\right)+\mathbb{D}\left(y, \frac{\partial \boldsymbol{W}}{\partial t}\right)\right)+\lambda_{0}(1-\chi(\boldsymbol{y}))(\mathbb{D}(x, \boldsymbol{w})+\mathbb{D}(y, \boldsymbol{W})), \\
\nabla \cdot \boldsymbol{w}=0,
\end{gathered}
$$

where

$$
\mathbb{D}(y, \boldsymbol{W})=\frac{1}{2}\left(\nabla_{y} \boldsymbol{W}+\left(\nabla_{y} \boldsymbol{W}\right)^{*}\right), \quad\langle U\rangle_{Z}=\int_{Z} U(\boldsymbol{y}) \mathrm{d} y .
$$

Lemma 3.4 For almost every $(\boldsymbol{x}, t) \in \Omega_{T}$ the limiting functions $\boldsymbol{w}, \boldsymbol{W}$ and $P$ satisfy in $Y \times(0, T)$ the system of microscopic equations

$$
\nabla_{y} \cdot \widetilde{\mathbb{P}}-\nabla_{y} P=0, \quad \nabla_{y} \cdot \boldsymbol{W}=0 .
$$

LEMma 3.5 The limiting stress tensor $\mathbb{P}^{(0)}$ has the form

$$
\mathbb{P}^{(0)}(\boldsymbol{w})=\mathbb{A}_{0}: \mathbb{D}\left(x, \frac{\partial \boldsymbol{w}}{\partial t}\right)+\mathbb{A}_{1}: \mathbb{D}(x, \boldsymbol{w})+\int_{0}^{t} \mathbb{A}_{2}(t-\tau): \mathbb{D}(x, \boldsymbol{w}(\boldsymbol{x}, \tau) \mathrm{d} \tau,
$$

where the fourth-rank constant tensors $\mathbb{A}_{0}, \mathbb{A}_{1}$, and the fourth-rank tensor $\mathbb{A}_{2}(t)$ are defined by formulas (3.7)-(3.9) below, and the tensor $\mathbb{A}_{0}$ is symmetric and strictly positive definite.

Proof. We just outline the scheme of the proof. It is enough to express $\mathbb{D}(y, \partial \boldsymbol{W} / \partial t)$ and $\mathbb{D}(y, \boldsymbol{W})$ through $\mathbb{D}(x, \partial \boldsymbol{w} / \partial t)$ and $\mathbb{D}(x, \boldsymbol{w})$ using equations (3.4). Namely, we look for the solution to the system (3.4) in the form

$$
\begin{gathered}
\boldsymbol{W}=\sum_{i, j=1}^{3} \int_{0}^{t} \boldsymbol{W}^{(i j)}(\boldsymbol{y}, t-\tau) Z_{i j}(\boldsymbol{x}, \tau) \mathrm{d} \tau, \\
P=\sum_{i, j=1}^{3} \int_{0}^{t} P^{(i j)}(\boldsymbol{y}, t-\tau) Z_{i j}(\boldsymbol{x}, \tau) \mathrm{d} \tau,
\end{gathered}
$$

where

$$
\mathbb{Z}=\mu_{0} \mathbb{D}\left(x, \frac{\partial \boldsymbol{w}}{\partial t}\right)-\lambda_{0} \mathbb{D}(x, \boldsymbol{w}), \quad Z_{i j}=\boldsymbol{e}_{i} \cdot\left(\mathbb{Z} \cdot \boldsymbol{e}_{j}\right),
$$

and $\left(e_{1}, e_{2}, e_{3}\right)$ are the standard Cartesian basis vectors. 
Then the functions $\boldsymbol{W}^{(i j)}$ and $P^{(i j)}$ satisfy in $Y \times(0, T)$ the periodic initial boundary value problems

$$
\left.\begin{array}{r}
\nabla_{y} \cdot\left(\chi \mu_{0} \mathbb{D}\left(y, \frac{\partial \boldsymbol{W}^{(i j)}}{\partial t}\right)+\lambda_{0}(1-\chi) \mathbb{D}\left(y, \boldsymbol{W}^{(i j)}\right)-P^{(i j)} \mathbb{I}\right)=0, \\
\nabla_{y} \cdot \boldsymbol{W}^{(i j)}=0, \\
\chi(\boldsymbol{y}) \boldsymbol{W}^{(i j)}(\boldsymbol{y}, 0)=\boldsymbol{W}_{0}^{(i j)}(\boldsymbol{y}),
\end{array}\right\}
$$

where $2 \mathbb{J}^{(i j)}=e_{i} \otimes e_{j}+e_{j} \otimes e_{i}$, and for any vectors $\boldsymbol{a}, \boldsymbol{b}$ and $\boldsymbol{c}$ the matrix $\boldsymbol{a} \otimes \boldsymbol{b}$ is defined by $(a \otimes b) \cdot c=a(b \cdot c)$.

Therefore,

$$
\begin{aligned}
& \left\langle\mathbb{D}\left(y, \frac{\partial \boldsymbol{W}}{\partial t}\right)\right\rangle_{Y_{f}}=\sum_{i, j=1}^{3}\left\langle\mathbb{D}\left(y, \boldsymbol{W}_{0}^{(i j)}\right)\right\rangle_{Y_{f}} Z_{i j}+\sum_{i, j=1}^{3} \int_{0}^{t}\left\langle\mathbb{D}\left(y, \frac{\partial \boldsymbol{W}^{(i j)}}{\partial \tau}\right)(\boldsymbol{y}, t-\tau)\right\rangle_{Y_{f}} Z_{i j}(\boldsymbol{x}, \tau) \mathrm{d} \tau \\
& =\sum_{i, j=1}^{3}\left(\left\langle\mathbb{D}\left(y, \boldsymbol{W}_{0}^{(i j)}\right)\right\rangle_{Y_{f}} \otimes \mathbb{J}^{(i j)}\right): \mathbb{Z} \\
& +\sum_{i, j=1}^{3} \int_{0}^{t}\left(\left\langle\mathbb{D}\left(y, \frac{\partial \boldsymbol{W}^{(i j)}}{\partial t}\right)(\boldsymbol{y}, t-\tau)\right\rangle_{Y_{f}} \otimes \mathbb{J}^{(i j)}\right): \mathbb{Z}(\boldsymbol{x}, \tau) \mathrm{d} \tau \\
& =\sum_{i, j=1}^{3}\left(\left\langle\mu_{0} \mathbb{D}\left(y, \boldsymbol{W}_{0}^{(i j)}\right)\right\rangle_{Y_{f}} \otimes \mathbb{J}^{(i j)}\right): \mathbb{D}\left(x, \frac{\partial \boldsymbol{w}}{\partial t}\right) \\
& -\lambda_{0} \sum_{i, j=1}^{3}\left(\left\langle\mathbb{D}\left(y, \boldsymbol{W}_{0}^{(i j)}\right)\right\rangle_{Y_{f}} \otimes \mathbb{J}^{(i j)}\right): \mathbb{D}(x, \boldsymbol{w}) \\
& +\mu_{0} \sum_{i, j=1}^{3} \int_{0}^{t}\left(\left\langle\mathbb{D}\left(y, \frac{\partial \boldsymbol{W}^{(i j)}}{\partial t}\right)(\boldsymbol{y}, t-\tau)\right\rangle_{Y_{f}} \otimes \mathbb{J}^{(i j)}\right): \mathbb{D}\left(x, \frac{\partial \boldsymbol{w}}{\partial \tau}\right)(\boldsymbol{x}, \tau) \mathrm{d} \tau \\
& -\lambda_{0} \sum_{i, j=1}^{3} \int_{0}^{t}\left(\left\langle\mathbb{D}\left(y, \frac{\partial \boldsymbol{W}^{(i j)}}{\partial t}\right)(\boldsymbol{y}, t-\tau)\right\rangle_{Y_{f}} \otimes \mathbb{J}^{(i j)}\right): \mathbb{D}(x, \boldsymbol{w})(\boldsymbol{x}, \tau) \mathrm{d} \tau, \\
& \langle\mathbb{D}(y, \boldsymbol{W})\rangle_{Y_{s}}=\sum_{i, j=1}^{3} \int_{0}^{t}\left\langle\mathbb{D}\left(y, \boldsymbol{W}^{(i j)}\right)(\boldsymbol{y}, t-\tau)\right\rangle_{Y_{f}} Z_{i j}(\boldsymbol{x}, \tau) \mathrm{d} \tau \\
& =\mu_{0} \sum_{i, j=1}^{3} \int_{0}^{t}\left(\left\langle\mathbb{D}\left(y, \boldsymbol{W}^{(i j)}\right)(\boldsymbol{y}, t-\tau)\right\rangle_{Y_{s}} \otimes \mathbb{J}^{(i j)}\right): \mathbb{D}\left(x, \frac{\partial \boldsymbol{w}}{\partial \tau}\right)(\boldsymbol{x}, \tau) \mathrm{d} \tau \\
& -\lambda_{0} \sum_{i, j=1}^{3} \int_{0}^{t}\left(\left\langle\mathbb{D}\left(y, \boldsymbol{W}^{(i j)}\right)(\boldsymbol{y}, t-\tau)\right\rangle_{Y_{s}} \otimes \mathbb{J}^{(i j)}\right): \mathbb{D}(x, \boldsymbol{w})(\boldsymbol{x}, \tau) \mathrm{d} \tau,
\end{aligned}
$$


and

$$
\begin{aligned}
& \mathbb{A}_{0}=m \sum_{i, j=1}^{3} J^{(i j)} \otimes J^{(i j)}+\mathbb{B}_{0}, \\
& \mathbb{A}_{1}=\lambda_{0}(1-m) \sum_{i, j=1}^{3} J^{(i j)} \otimes J^{(i j)}-\lambda_{0} \mathbb{B}_{0}+\mu_{0} \mathbb{B}_{1}(0), \\
& \mathbb{A}_{2}=\mu_{0} \frac{\mathrm{d}}{\mathrm{d} t} \mathbb{B}_{1}(t)-\lambda_{0} \mathbb{B}_{1}(t),
\end{aligned}
$$

where

$$
\begin{aligned}
\mathbb{B}_{0} & =\sum_{i, j=1}^{3}\left\langle\mu_{0} \mathbb{D}\left(y, \boldsymbol{W}_{0}^{(i j)}\right)\right\rangle_{Y_{f}} \otimes \mathbb{J}^{(i j)}, \\
\mathbb{B}_{1}(t) & =\sum_{i, j=1}^{3}\left(\frac{\mathrm{d}}{\mathrm{d} t} \mu_{0}\left\langle\mathbb{D}\left(y, \boldsymbol{W}^{(i j)}\right)\right\rangle_{Y_{f}}(t)+\lambda_{0}\left\langle\mathbb{D}\left(y, \boldsymbol{W}^{(i j)}\right)\right\rangle_{Y_{s}}(t)\right) \otimes \mathbb{J}^{(i j)} .
\end{aligned}
$$

\section{Conclusions}

In the present paper we have shown how to model some physical processes using modern methods of the mathematical analysis. We started with the free boundary problem for a joint motion of two immiscible incompressible fluids on the microscopic level. Theoretically this mathematical model is the most suitable; however it has no practical value, because we have to solve the problem in the physical scale of several hundred meters, while the coefficients oscillate on the physical scale of some microns. The practical value of the model appears only after homogenization. In turn, the homogenization has at least three levels approximation, which depend on the dimensionless criteria of the physical problem. The first level approximation is the well-known Muskat problem. The second level is the Muskat-Terzaghi-Biot problem. Finally, the third level approximation of the free boundary problem on the microscopic level is the Muskat problem for viscoelastic filtration.

Under certain conditions on the dimensionless criteria of the physical problem we have proved that solutions of the free boundary problem for a joint motion of two immiscible incompressible fluids on the microscopic level converge to the solution of the Muskat problem for viscoelastic filtration as the small parameter of the homogenization tends to zero.

\section{Acknowledgements}

This research is partially supported by the Federal Program "Research and scientific-pedagogical brainpower of Innovative Russia” for 2009-2013 (State Contract 02.740.11.0613).

\section{REFERENCES}

1. Biot, M. General theory of three dimensional consolidation. J. Appl. Phys. 12 (1941), 155-164. JFM 67.0837.01

2. Burridge, R., \& Keller, J. B. Poroelasticity equations derived from microstructure. J. Acoustic Soc. Amer. 70 (1981), 1140-1146. Zbl 0519.73038 
3. CONCA, C. On the application of the homogenization theory to a class of problems arising in fluid mechanics. J. Math. Pures Appl. 64 (1985), 31-75. Zbl 0566.35080 MR 0802383

4. KIRK, W. A., Sims, B. (eds.) Handbook of Metric Fixed Point Theory. Kluwer, London (2001). Zbl 0970.54001 MR 1904271

5. LAdyZhenskaya, O. A. The Mathematical Theory of Viscous Incompressible Flow. Gordon and Breach, New York (1969). Z Zbl 0184.52603 MR 0254401

6. Ladyzhenskaya, O. A., Solonnikov, V. A., \& Ural'tseva, N. N. Linear and Quasilinear Equations of Parabolic Type. Amer. Math. Soc., Providence, RI (1968). Z Zbl 0174.15403 MR 0244627

7. LiONs, J.-L. Quelques méthodes de résolution des problèmes aux limites non linéaires. Dunod and Gauthier-Villars, Paris (1969). Z Zbl 0189.40603 MR 0259693

8. Meirmanov, A. Nguetseng's two-scale convergence method for filtration and seismic acoustic problems in elastic porous media. Siberian Math. J. 48 (2007), 519-538. Zbl 1164.74393 MR 2347913

9. Muskat, M. Two-fluid system in porous media. The encroachment of water into an oil sand. Physics $\mathbf{5}$ (1934), 250-264. JFM 60.1388.01

10. Nguetseng, G. A general convergence result for a functional related to the theory of homogenization. SIAM J. Math. Anal. 20 (1989), 608-623. Zbl 0688.35007 MR 0990867

11. Nguetseng, G. Asymptotic analysis for a stiff variational problem arising in mechanics. SIAM J. Math. Anal. 21 (1990), 1394-1414. Zbl 0723.73011 MR 1075584

12. RAdKeVich, E. On the spectrum of the pencil in the Verigin-Muskat problem. Sbornik Math. 80 (1995), 33-73. Zbl 0820.35147 MR 1257336

13. SÁnchez-Palencia, E. Non-Homogeneous Media and Vibration Theory. Lecture Notes in Phys. 127, Springer (1980). Z Zbl 0432.70002 MR 0578345

14. Siegel, M., Caflisch, R. E., \& Howison, S. Global existence, singular solutions, and ill-posedness for the Muskat problem. Comm. Pure Appl. Math. 57 (2004), 1374-1411. Zbl 1062.35089 MR 2070208

15. Terzaghi, K. Die Berechnung der Durchlassigkeitsziffer des Tones aus dem Verlauf der hydrodynamischen Spannungserscheinungen. Sitzungsber. Akad. Wiss. Wien Math.-Naturwiss. Kl. 132 (1923), 104-124.

16. YI, F. Global classical solution of Muskat free boundary problem. J. Math. Anal. Appl. 288 (2003), 442-461. Zbl 1038.35083 MR 2019452 